\title{
SHINING, A Survey of Far-infrared Lines in Nearby Galaxies. II. Line-deficit Models, AGN Impact, [C II]-SFR Scaling Relations, and Mass-Metallicity Relation in (U)LIRGs
}

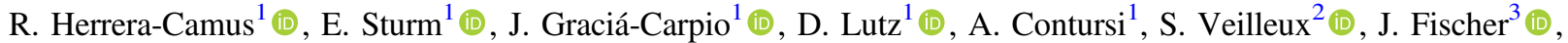

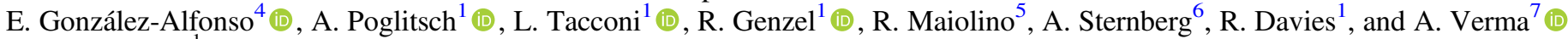 \\ ${ }^{1}$ Max-Planck-Institut für Extraterrestrische Physik (MPE), Giessenbachstr., D-85748 Garching, Germany; rhc@ mpe.mpg.de \\ ${ }^{2}$ Department of Astronomy and Joint Space-Science Institute, University of Maryland, College Park, MD 20742, USA \\ ${ }^{3}$ Naval Research Laboratory, Remote Sensing Division, 4555 Overlook Avenue SW, Washington, DC 20375, USA \\ ${ }^{4}$ Departamento de Física y Matemáticas, Universidad de Alcalá, Campus Universitario, E-28871 Alcalá de Henares, Madrid, Spain \\ ${ }^{5}$ Kavli Institute for Cosmology, University of Cambridge, Madingley Road, Cambridge CB3 OHA, UK \\ ${ }^{6}$ Raymond and Beverly Sackler School of Physics and Astronomy, Tel Aviv University, Ramat Aviv 69978, Israel \\ ${ }_{7}$ Oxford University, Dept. of Astrophysics, Oxford OX1 3RH, UK \\ Received 2018 February 19; revised 2018 March 4; accepted 2018 March 10; published 2018 July 10
}

\begin{abstract}
The SHINING survey offers a great opportunity to study the properties of the ionized and neutral media of galaxies from prototypical starbursts and active galactic nuclei (AGNs) to heavily obscured objects. Based on Herschel/ PACS observations of the main far-infrared (FIR) fine-structure lines, in this paper, we analyze the physical mechanisms behind the observed line deficits in galaxies, the apparent offset of luminous infrared galaxies (LIRGs) from the mass-metallicity relation, and the scaling relations between [C II] $158 \mu \mathrm{m}$ line emission and star formation rate (SFR). Based on a toy model and the Cloudy code, we conclude that the increase in the ionization parameter with FIR surface brightness can explain the observed decrease in the line-to-FIR continuum ratio of galaxies. In the case of the [C II] line, the increase in the ionization parameter is accompanied by a reduction in the photoelectric heating efficiency and the inability of the line to track the increase in the FUV radiation field as galaxies become more compact and luminous. In the central approximately kiloparsec regions of AGN galaxies, we observe a significant increase in the $[\mathrm{O} \mathrm{I}] 63 \mu \mathrm{m} /[\mathrm{C}$ II] line ratio; the AGN impact on the line-to-FIR ratios fades on global scales. Based on extinction-insensitive metallicity measurements of LIRGs, we confirm that they lie below the mass-metallicity relation, but the offset is smaller than those reported in studies that use optical-based metal abundances. Finally, we present scaling relations between [C II] emission and SFR in the context of the main sequence of star-forming galaxies.
\end{abstract}

Key words: galaxies: abundances - galaxies: active - galaxies: general - galaxies: ISM - galaxies: starburst galaxies: star formation

\section{Introduction}

The far-infrared (FIR) fine-structure lines of $\mathrm{C}, \mathrm{N}$, and $\mathrm{O}$ offer a powerful tool to characterize the interstellar medium (ISM) of nearby and high- $z$ galaxies. In this context, the SHINING survey of galaxies ("Survey with Herschel of the ISM in Nearby INfrared Galaxies"; PI Sturm) was planned with the purpose of obtaining a comprehensive view of the physical processes at work in the ISM of galaxies, ranging from moderately star-forming to the most dense and obscured environments in luminous infrared galaxies (LIRGs) and around active galactic nuclei (AGNs). For this, we used Herschel PACS to observe the six main FIR atomic and ionic lines in the $\sim 55-200 \mu \mathrm{m}$ range. The description of the survey and the main general results are presented in Paper I (HerreraCamus et al. 2018).

One of the open questions in the study of the ISM of galaxies is what are the physical mechanisms that drive the decrease in the line-to-FIR continuum ratios - commonly referred to as "line deficit" - in the most dense, energetic galactic environments. In the case of the brightest of the FIR fine-structure lines, the ionized carbon line [C II] at $157.74 \mu \mathrm{m}$, the [C II]/FIR continuum ratio typically range from $\sim 10^{-2}$ in normal, starforming galaxies to $\sim 10^{-4}$ in galaxies with a buried, compact, luminous nucleus (e.g., Malhotra et al. 1997, 2001; Luhman et al. 1998, 2003; Graciá-Carpio et al. 2011; Díaz-Santos et al. 2013; Farrah et al. 2013; González-Alfonso et al. 2015; Ibar et al. 2015; Contursi et al. 2017; Smith et al. 2017; HerreraCamus et al. 2018).

Several physical effects have been suggested to explain the [C II] deficit, including the reduction of the photoelectric heating efficiency due to the charging or destruction of the small dust grains (Malhotra et al. 2001; Croxall et al. 2012); high-ionization parameters in ionized regions that cause an increase in the fraction of UV photons absorbed by dust relative to the UV photons available to ionized and excite the neutral gas (Luhman et al. 2003; Abel et al. 2009; GraciáCarpio et al. 2011; Fischer et al. 2014); the impact of AGNs on the ionization state of the gas (Sargsyan et al. 2012; Langer \& Pineda 2015); strong continuum extinction at $158 \mu \mathrm{m}$; the saturation of the upper energy level at gas temperatures higher than the excitation temperature of the line (Stacey et al. 2010; Muñoz \& Oh 2015); dense PDRs with gas densities higher than the critical density of the [C II] line, among others. One of the main goals of this paper is to use the sophisticated theoretical models available (e.g., Cloudy, PDR Toolbox; Kaufman et al. 2006; Pound \& Wolfire 2008; Ferland et al. 2013; Fischer et al. 2014 ) to interpret the line-to-FIR continuum trends and understand what the main physical processes are behind the observed line deficits in galaxies.

The detailed characterization of the FIR fine-structure lines in nearby galaxies is also very relevant in the current era of sensitive interferometers, such as ALMA and NOEMA. These 
observatories have made possible detections and spatially resolved observations of galaxies from the epoch of the peak of cosmic star formation to the era of reionization in the restframe FIR lines. [C II] $158 \mu \mathrm{m}$ (e.g., De Breuck et al. 2014; Riechers et al. 2014; Capak et al. 2015; Inoue et al. 2016; Pentericci et al. 2016), [N II] $122 \mu \mathrm{m}$ (e.g., Ferkinhoff et al. 2015; Pavesi et al. 2016), and [O III] $88 \mu \mathrm{m}$ (e.g., Nagao et al. 2012; Carniani et al. 2017), have been detected in galaxies ranging from typical, star-forming galaxies to extreme starbursts. In this paper, we present scaling relations between the [C II] luminosity and the star formation rate (SFR) as a function of various galaxy properties (separation from the main sequence of galaxies, star formation efficiency, FIR surface brightness, galaxy type), which can be useful for the interpretation of the growing number of high- $z$ galaxies detected in [C II] line emission.

Finally, another strong asset of the FIR lines is their ability to penetrate extremely high dust column densities and characterize the conditions in the most obscured objects. ${ }^{8}$ One immediate application is to use the FIR lines to determine the metal abundance of LIRGs (e.g., Nagao et al. 2011; FernándezOntiveros et al. 2016; Pereira-Santaella et al. 2017). LIRGs are believed to lie below the well-known mass-metallicity relation for star-forming galaxies (e.g., Tremonti et al. 2004; Rupke et al. 2008; Kilerci Eser et al. 2014); though, these results are based on optical-based metallicity measurements. One possibility is that the gas in LIRGs has indeed lower metal content compared to normal galaxies with similar stellar masses. The reason is the accretion of low-metallicity gas-triggered by their history of interactions-from the outskirts toward the central regions (Rupke et al. 2010; Torrey et al. 2012). The other alternative is that the optical-based metallicity measurements in LIRGs are underestimated due to strong dust extinction. The spectral coverage and unprecedented sensitivity offered by Herschel provide us now with the opportunity to use FIR lines as extinction-free metallicity tracers and to test which of the two scenarios described above is responsible for the observed offset of LIRGs from the mass-metallicity relation.

This paper is organized as follows. In Section 2, we give a brief introduction to the SHINING sample of galaxies. In Section 3, we analyze the observed line-to-continuum trends using a toy model (Section 3.1) and the Cloudy code (Section 3.2). We investigate the influence of AGN emission on the [C II] and [O I] $63 \mu \mathrm{m}$ line-to-FIR ratios in Section 3.3. We present scaling relations between the [C II] line emission and the SFR in Section 3.4. Finally, we revisit the observed mass-metallicity relation in (U)LIRGs, this time using the [O III] $88 \mu \mathrm{m} /[\mathrm{N} \mathrm{III]} 57 \mu \mathrm{m}$ line ratio as an extinction-insensitive metallicity tracer (Section 3.5). We present our summary and conclusions in Section 4.

\section{Galaxy Sample}

A detailed description of the SHINING sample characteristics, observational strategy, data reduction, and flux measurements can be found in Paper I (Herrera-Camus et al. 2018). Here we provide a brief summary of these topics.

The SHINING sample consists of 52 nearby $(z<0.2)$ galaxies that were observed with the PACS spectrometer

\footnotetext{
Note, however, that in extreme dust environments, such as that found in Arp 220, the dust optical depth is $\gtrsim 1$ in the far-infrared wavelength range up to $\sim 850 \mu \mathrm{m}$ (e.g., Sakamoto et al. 2008; Rangwala et al. 2011).
}

(Poglitsch et al. 2010) on board Herschel (Pilbratt et al. 2010). The breakdown by galaxy type is: 8 star-forming, 23 AGNs, and 21 (U)LIRGs. SHINING includes some archetypical galaxies, such as M82, M83, NGC 253, NGC 1068, Circinus, and Arp 220.

The survey includes observations of the six ionized and PDR lines in the 55-200 $\mu \mathrm{m}$ range. These are [C II] $158 \mu \mathrm{m}$, [O I] $145 \mu \mathrm{m},[\mathrm{N} \mathrm{II}] 122 \mu \mathrm{m}$, [O III] $88 \mu \mathrm{m},[\mathrm{O}$ I] $63 \mu \mathrm{m}$, and $[\mathrm{N} \mathrm{III}] 57 \mu \mathrm{m}$. The data were reduced using HIPE v13.0 (Herschel Interactive Processing Environment"; Ott 2010). In the case of galaxies that were considered point sources for Herschel (the PACS spectrometer point-spread function is $\sim 6^{\prime \prime}-11^{\prime \prime}$ in the instrument wavelength range), we applied a point-source correction determined both theoretically and from dedicated PV observations (Poglitsch et al. 2010). The integrated line and continuum fluxes for all the SHINING sources are listed in Paper I (Table 6 and 7; Herrera-Camus et al. 2018).

In addition to the SHINING galaxies, in this paper, we include a compilation of previous ISO extragalactic observations taken from the literature (Malhotra et al. 2001; Negishi et al. 2001; Luhman et al. 2003; Lutz et al. 2003; Brauher et al. 2008), and PACS [C II] observations of local starburst, (U)LIRGs, and AGNs by Sargsyan et al. (2012) and Farrah et al. (2013). The FIR sizes for the SHINING and the ancillary galaxies were drawn from Lutz et al. (2016) and were derived from a two-dimensional Gaussian fit to the $70 \mu \mathrm{m}$ image of the galaxy, with PSF width subtracted in quadrature. We also include in our analysis high- $z(z>4)$ galaxies detected in [C II] line and continuum emission and with size measurements available (Walter et al. 2012; Riechers et al. 2013; De Breuck et al. 2014; Capak et al. 2015).

Throughout the paper, far-infrared luminosities were measured using the definition given in Helou et al. (1988):

$$
\begin{aligned}
& F_{\text {FIR }}(42.5 \mu \mathrm{m}-122.5 \mu \mathrm{m})=1.26 \times 10^{-14} \\
& \quad \times\left(2.58 S_{60 \mu \mathrm{m}}+S_{100 \mu \mathrm{m}}\right) .
\end{aligned}
$$

\section{Analysis}

In Paper I, we show how the line-to-FIR ratios of galaxies decrease as a function of FIR luminosity, star formation efficiency, and FIR surface brightness. In the case of the [C II] and [O I] lines, the dispersion in the relation between the lineto-FIR ratio and $\Sigma_{\text {FIR }}$ is only $\sim 0.3$ dex over almost five orders of magnitude in $\Sigma_{\mathrm{FIR}}$ (see also Lutz et al. 2016; Díaz-Santos et al. 2017). With the purpose of understanding the existence and tightness of these correlations, in this section, we present an analysis based on a toy model powered by the PDR toolbox (Section 3.1) and the Cloudy code (Section 3.2).

\subsection{A Toy Model to Explore the PDR Line Deficit as a Function of $\Sigma_{F I R}$}

We construct a toy model with the aim of exploring what drives the observed decline of the ratio between the PDR lines ([C II], and [O I] 63 and $145 \mu \mathrm{m}$ ) and the FIR continuum emission as a function of $\Sigma_{\mathrm{FIR}}$. Based on the work by Wolfire et al. (1990), we consider two "extreme" scenarios for the distribution of stars and gas clouds in galaxies. These scenarios

\footnotetext{
9 HIPE is a joint development by the Herschel Science Ground Segment Consortium, consisting of ESA, the NASA Herschel Science Center, and the HIFI, PACS, and SPIRE consortia.
} 
set characteristic values for the physical conditions in the ISM of the galaxy, including the density of the neutral gas clouds $\left(n_{\mathrm{H}}\right)$, the FUV radiation field intensity impinging upon $\left(G_{0}\right)$, and the FIR continuum and line intensity they emit. The goal is to explore what is driving the line deficits as galaxies become more compact and/or luminous.

\subsubsection{Scenario 1: Dense PDR}

In this scenario, we assume that $O B$ stars are closely associated with molecular clouds. In this case, the incident FUV flux on the molecular clouds is dominated by the nearest stars, and $G_{0}$ can be approximated as:

$$
\left\langle G_{0}\right\rangle^{\mathrm{OB}} \simeq 10^{-2}\left(\frac{L_{*}}{L_{\odot}}\right)\left(\frac{r}{1 \mathrm{pc}}\right)^{-2},
$$

where $L_{*}$ is the average stellar or OB association FUV luminosity, and $r$ is the distance from the stars to the molecular cloud.

Consider now the idealized problem of a static, spherically symmetric equilibrium $\mathrm{H}$ II region ionized by a point source as discussed by Draine (2011). If the product of the rate of ionizing photons $\left(Q_{0}\right)$ and the rms density of the ionized gas $\left(n_{\mathrm{rms}}\right)$ is $\left(Q_{0} / 10^{49} \mathrm{~s}^{-1}\right) n_{\mathrm{rms}} \gg 10^{2} \mathrm{~cm}^{-3}$, then the radiation pressure acts to concentrate the gas in a spherical shell. This implies that the pressure of ionized gas at the edge of the $\mathrm{H}$ II region is higher than that obtained in the uniform-density $\mathrm{H}$ II region approximation. Conversely, the external pressure confining the $\mathrm{H}$ II region has to be higher.

For our calculation of the PDR line-to-continuum ratio, we start with a stellar cluster with an ionizing photon rate $Q_{0}=10^{52} \mathrm{~s}^{-1}$, which is typical for giant $\mathrm{H}$ II regions such as 30 Dor (Kennicutt 1991). Based on $Q_{0}$, we estimate the stellar cluster FUV luminosity following $L_{\mathrm{FUV}}=5.3 \times 10^{5}\left(Q_{0} / 10^{49} \mathrm{~s}\right) L_{\odot}$ (Kaufman et al. 2006). Then, for a given $\mathrm{H}$ II region radius $R$, we determine $p_{\text {edge }}$ using the Draine (2011) model calculations (e.g., see the model grid results in Figure 11 of Draine 2011). We use a standard model that adopts values of $\beta=3$ and $\gamma=10$, where $\beta$ is the ratio of the power in non-ionizing photons to the power in photons with $h \nu>13.6 \mathrm{eV}$, and $\gamma$ is a dimensionless parameter that depends on the gas temperature and the mean ionizing photon energy.

We then solve for $n_{\mathrm{H}}$ by equating the ionized gas pressure at the edge of the $\mathrm{H}$ II region with the pressure of the confining neutral gas, i.e.,

$$
n_{\mathrm{H}}(r=R) \simeq \frac{p_{\text {edge }}}{k T_{\mathrm{PDR}}}
$$

For the PDR temperature, we assume $T_{\mathrm{PDR}}=500 \mathrm{~K},{ }^{10}$ consistent with observed temperatures in Galactic PDRs (Hollenbach \& Tielens 1999; Sheffer et al. 2011).

Now that we have expressions for $\left\langle G_{0}\right\rangle^{\mathrm{OB}}$ and $n_{\mathrm{H}}$ as a function of $R$, we consider a family of $\mathrm{H}$ II region/molecular cloud complexes with sizes that range from $R=2 \mathrm{pc}$ to $100 \mathrm{pc}$, and for each size we use the PDR Toolbox (Kaufman et al. 2006; Pound \& Wolfire 2008) to compute the intensity of the [C II], [O I] $63 \mu \mathrm{m}$, and [O I] $145 \mu \mathrm{m}$ lines (in units of erg cm${ }^{-2} \mathrm{~s}^{-1} \mathrm{sr}^{-1}$ ). Following Kaufman et al. (1999), we also calculate the FIR dust continuum intensity in the optically

\footnotetext{
${ }^{10}$ Assuming $T_{\mathrm{PDR}}=300 \mathrm{~K}$ instead of $500 \mathrm{~K}$ produces very similar results. Assuming $T_{\mathrm{PDR}}=1000 \mathrm{~K}$ results in $[\mathrm{C} \mathrm{II}] / \mathrm{FIR}$ ratios about $50 \%$ lower for $\Sigma_{\mathrm{FIR}} \lesssim 10^{10} L_{\odot} \mathrm{kpc}^{-2}$
}

thin limit as $I_{\mathrm{FIR}}=2 \times 1.3 \times 10^{-4}\left\langle G_{0}\right\rangle^{\mathrm{OB}}\left[\mathrm{erg} \mathrm{cm}^{-2} \mathrm{~s}^{-1} \mathrm{sr}^{-1}\right]$ (recall that $G_{0}$ corresponds to $1.6 \times 10^{-3} \mathrm{erg} \mathrm{cm}^{-2} \mathrm{~s}^{-1}$ and $\left.1.3 \times 10^{-4}=1.6 \times 10^{-3} / 4 \pi\right)$, although we note that the optically thin limit assumption is not accurate for Arp 220like environments characterized by extinction of the FIR lines due to dust and high column densities of $\mathrm{OH}$ and $\mathrm{H}_{2} \mathrm{O}$ (González-Alfonso et al. 2004, 2012, 2015). Finally, we calculate the FIR surface brightness as $\Sigma_{\mathrm{FIR}} \simeq 2.5 \times$ $10^{9} \times 4 \pi I_{\text {FIR }} \times \Phi_{\mathrm{A}}\left[L_{\odot} \mathrm{kpc}^{-2}\right]$, where $\Phi_{\mathrm{A}}$ is the beam area filling factor of the PDR regions. When $\Phi_{\mathrm{A}}<1$, the area of [C II] emitting sources does not fill the beam, while $\Phi_{\mathrm{A}}>1$ means that the PDR surface area intercepted by the beam exceeds the beam projected area.

\subsubsection{Scenario 2: Clouds irradiated by an average ISRF}

In this scenario, we consider that $\mathrm{OB}$ stars are randomly placed with respect to the clouds. In this case, the FUV flux incident on clouds is dominated by the average interstellar radiation field (ISRF). Following Wolfire et al. (1990), we can express $G_{0}$ in this particular scenario as:

$$
\begin{aligned}
\left\langle G_{0}\right\rangle^{\mathrm{ISRF}} \simeq & 3 \times 10^{4}\left(\frac{L_{\mathrm{FIR}}}{10^{10} L_{\odot}}\right) \times\left(\frac{\lambda}{100 \mathrm{pc}}\right) \\
& \times\left(\frac{100 \mathrm{pc}}{S}\right)^{3}\left[1-e^{-S / \lambda}\right],
\end{aligned}
$$

where $S$ is the radius of the IR emitting region and $\lambda$ is the mean cloud separation along the line of sight.

For the neutral gas volume density of the surface of the clouds illuminated by $\left\langle G_{0}\right\rangle^{I S R F}$, we assume values of $10^{2}$ and $10^{3} \mathrm{~cm}^{-3}$, which represent the range of observed densities in our Galaxy and extragalactic sources (e.g., Stacey et al. 1985; Wolfire et al. 2010; Graciá-Carpio et al. 2011; Pineda et al. 2013). Then, assuming an IR source with a luminosity of $L_{\mathrm{IR}}=5 \times 10^{10} L_{\odot}$, and varying the radius of the IR emitting source from $S=0.1$ to $2 \mathrm{kpc}$ (which produces FIR surface brightnesses in the $\Sigma_{\text {FIR }} \sim$ $10^{9}-10^{12} L_{\odot} \mathrm{kpc}^{-2}$ range), we calculate $\left\langle G_{0}\right\rangle^{\mathrm{ISRF}}$, assuming that the mean separation between clouds is twice the mean separation between stars (i.e., $\lambda=2 d_{*} \approx 2 \times\left[\left(L_{\mathrm{FIR}} / L_{*}\right) / \frac{4}{3} \pi S^{3}\right]^{-1 / 3}$; for the luminosity of the star cluster, we assume $L_{*}=5 \times 10^{5} L_{\odot}$, the typical value for $\mathrm{OB}$ associations in clouds such as Orion or M17). Finally, for each size $S$ and its corresponding $\left\langle G_{0}\right\rangle^{\text {ISRF }}$ value, we use the PDR Toolbox to calculate the intensity of the [C II] and [O I] lines for assumed characteristic densities of $n_{\mathrm{H}}=10^{2}$ and $10^{3} \mathrm{~cm}^{-3}$ (e.g., Malhotra et al. 2001; Parkin et al. 2013; Contursi et al. 2017).

As a final note on the model, calculations in the PDR Toolbox code assume a grain photoelectric heating rate, such as that derived by Bakes \& Tielens (1994). This rate includes a size distribution of particles extending from large grains $(\sim 0.25 \mu \mathrm{m})$ to small $(\sim 5 \AA)$ PAHs and explicitly accounts for the microphysics of small particles. In the numerical calculations of Bakes \& Tielens (1994), the grain photoelectric heating efficiency, $\epsilon_{\mathrm{ph}}$, is a function of the charging parameter, $\gamma=G_{0} T^{1 / 2} / n_{\mathrm{e}}$. We calculate $\gamma$ using $T$ and $G_{0}$ given by our assumption of the model scenario. For the electron density, we use the analytic expression presented in Röllig et al. (2006). ${ }^{11}$

\footnotetext{
11 The analytic expression in Röllig et al. (2006) for the electron density is $n_{\mathrm{e}} \approx 0.84 \times 10^{-4} \times n Z\left(1+\left(1+14.4 T^{0.75} /\left(n Z^{2}\right)\right)^{1 / 2}\right) \mathrm{cm}^{-3}$. The value of $T$ and $n$ are given by our model, and we assume gas with solar metallicity.
} 


\subsubsection{Toy Model Results and Comparison to Observations}

When comparing the line-to-dust continuum ratios observed in galaxies to those calculated by our model, we need to consider that the latter does not include contributions to the line emission by the ionized phase. This is of particular importance for the [C II] line that can arise from both the ionized and the neutral gas. One observational tool to constrain the fraction of the [C II] emission emitted by the neutral gas, $f_{[\mathrm{C} \text { II }]}^{\text {netal }}$, is to compare the $[\mathrm{C} \mathrm{II}]$ to one of the $[\mathrm{N} \mathrm{II}]$ lines. As discussed in Paper I (Herrera-Camus et al. 2018), based on the [C II]/ [N II] $122 \mu \mathrm{m}$ ratio, we find that $f_{\text {[C II] }}^{\text {neutal }}$ increases from $\sim 60 \%$ to $90 \%$ in the $S_{63 \mu \mathrm{m}} / S_{122 \mu \mathrm{m}} \sim 0.1-2$ range.

The neutral gas components contributing to the [C II] emission are PDRs, diffuse atomic gas, and "CO-faint" molecular gas (i.e., molecular $\mathrm{H}_{2}$ gas that resides in parts of clouds where $\mathrm{CO}$ has been dissociated). For the close association of stars and clouds, we assume that the [C II] emission is dominated by the PDRs and "CO-faint" molecular gas as indicated by the analysis of normal and starburst galaxies (e.g., Kaufman et al. 2006; Stacey et al. 2010; Croxall et al. 2012; Pineda et al. 2014). This is also the case for the [O I] lines, as standard models of starlight heating indicate that diffuse gas is too cool to emit [O I].

Figure 1 shows the comparison between our model outputs and the observed [C II]-to-FIR ratio as a function of $\Sigma_{\text {FIR }}$. We have scaled the model outputs by the inverse of $f_{[\mathrm{C} \mathrm{II}]}^{\text {neutral }}$ in order to account for the contribution from the ionized gas to the [C II] emission. We calculate $f_{\text {[C II] }}^{\text {neutral }}$ using a parameterization of this fraction as a function of $\Sigma_{\text {FIR }}$ (Croxall et al. 2017; Díaz-Santos

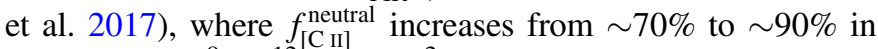
the $\Sigma_{\mathrm{FIR}} \sim 10^{9}-10^{12} \stackrel{[\mathrm{CII}}{L_{\odot}} \mathrm{kpc}^{-2}$ range.

The red curve shows the model result for the closeassociation model, assuming beam filling factors of $\Phi_{A}=$ $0.1,0.3$. and 1. Starburst galaxies and LIRGs typically have beam filling factors in the $\Phi_{A} \simeq 10^{-2}-1$ range; starburst galaxies with $\Sigma_{\mathrm{FIR}} \gtrsim 10^{11} L_{\odot} \mathrm{kpc}^{-2}$ tend to have beam filling factors approaching unity (e.g., Díaz-Santos et al. 2017). In the dense PDR scenario, as $\mathrm{H}$ II regions become more compact $G_{0}$ increases as $R^{-2} ; n_{\mathrm{H}}$ also increases but at a slightly lower rate. If the cloud density $n_{\mathrm{H}}$ is lower than the critical density $\left(n_{\text {crit }}\right)$ of the [C II] line-which in our model occurs when $\Sigma_{\text {FIR }} \lesssim$ $3 \times 10^{10} L_{\odot} \mathrm{kpc}^{-2}$ - then the intensity of [C II] emission is nearly independent of $G_{0}$ and only increases proportional to $n_{\mathrm{H}}$ (Kaufman et al. 1999). This implies that as H II regions become more compact, the FIR dust continuum intensity $\left(\propto G_{0}\right)$ increases at a faster rate than the [C II] intensity $\left(\propto n_{\mathrm{H}}\right)$, causing the [C II]/FIR ratio to decrease as a function of increasing $\Sigma_{\text {FIR }}$. Once the gas density of the neutral gas cloud confining the H II region reaches the critical density of the [C II] transition at around $\Sigma_{\mathrm{FIR}} \sim 3 \times 10^{10} L_{\odot} \mathrm{kpc}^{-2}$ (for $\Phi=1$ ), the intensity of [C II] emission becomes independent of both $G_{0}$ and $n_{\mathrm{H}}$, resulting in the decrease of the [C II]/FIR ratio with $\Sigma_{\mathrm{FIR}}$ at an even faster rate. In terms of the PDR mass-which is proportional to $N_{\mathrm{C}^{+}} R^{2}$, as the size of the $\mathrm{H}$ II region decreases $N_{\mathrm{C}^{+}}$remains constant but $G_{0}$, and consequently $\Sigma_{\mathrm{FIR}}$, increases resulting in lower PDR masses available to produce [C II] emission (see also González-Alfonso et al. 2008, 2015).

One additional factor in the dense PDR scenario that can contribute to the decrease of the [C II]-to-FIR ratio is the competition for the available UV photons between dust, ionized gas, and neutral hydrogen. The expectation is that as the ionization parameter $U$ increases, the fraction of $\mathrm{UV}$ photons that ionize and excite the gas is reduced by dust absorption, which results in enhanced FIR continuum emission compared to the intensity of the FIR lines (e.g., Voit 1992; Luhman et al. 2003; Abel et al. 2009; Graciá-Carpio et al. 2011; Fischer et al. 2014). In the Draine (2011) model of dusty $\mathrm{H}$ II regions, the compression of the ionized gas into a shell limits the ionization parameter at the half-ionization radius to $U \approx 0.01$ (see also Dopita et al. 2002; Stern et al. 2014). This value corresponds to the threshold where we expect dust to start absorbing an important fraction of the incident ionizing radiation (e.g., Graciá-Carpio et al. 2011), thus reducing the line-to-FIR ratio. This, in conjunction with the density effect described above, may also contribute to the decrease in the [C II]-to-FIR ratio as a function of $\Sigma_{\mathrm{FIR}}$ in the dense PDR scenario.

Finally, in the dense PDR scenario, the drop of the [C II]/FIR ratio as a function of $\Sigma_{\mathrm{FIR}}$ is not a result of the decrease in the photoelectric heating efficiency. In the $\Sigma_{\mathrm{FIR}} \sim 10^{9}-10^{12} L_{\odot} \mathrm{kpc}^{-2}$ range, the charging parameter increases from $\gamma \sim 1 \times 10^{4}$ to $\sim 8 \times 10^{4} \mathrm{~K}^{1 / 2} \mathrm{~cm}^{-3}$, which implies only a modest reduction in the $[\mathrm{C} \mathrm{II}] /$ FIR ratio of a factor of $\sim 4$. These values of $\gamma$ are consistent with those found in the H II/PDR complexes such as NGC 7023, Mon R2, and Ced 201 (Okada et al. 2013).

In the second scenario, we consider an average ISRF $\left\langle G_{0}\right\rangle^{\text {ISRF }}$ illuminating neutral clouds with densities that vary from $n_{\mathrm{H}}=10^{3} \mathrm{~cm}^{-3}$ (thin blue line) to $10^{3} \mathrm{~cm}^{-3}$ (thick blue line). Around $\Sigma_{\mathrm{FIR}} \approx 10^{9} L_{\odot} \mathrm{kpc}^{-2}$, the average ISRF is $\left\langle G_{0}\right\rangle^{\text {ISRF }} \approx 6$ and the efficiency of gas heated by photoelectric heating is high ([C II]/FIR 3\%), but still in the range of values observed in systems such as low-metallicity galaxies (e.g., Cigan et al. 2016). Now, as we make galaxies more compact, the average ISRF starts to increase, and at around $\Sigma_{\mathrm{FIR}} \approx 10^{10} L_{\odot} \mathrm{kpc}^{-2}$ reaches $\left\langle G_{0}\right\rangle^{\backslash \mathrm{SRF}} \approx 10^{2}$. From this point on, and if we keep $n_{\mathrm{H}}$ fixed (at values lower than $n_{\text {crit }}$ ), the intensity of [C II] becomes nearly independent of $G_{0}$ as this transition saturates at gas temperatures above $92 \mathrm{~K}$ (i.e., increases in $T$ do not appreciably change the intensity) and proportional to $n_{\mathrm{H}}$. At $\Sigma_{\mathrm{FIR}} \approx 3 \times 10^{11} L_{\odot} \mathrm{kpc}^{-2}$, the mean separation between clouds according to the model is $\lambda \approx 10 \mathrm{pc}$, and the [C II]-to-FIR ratio is $\approx 10^{-3}$. These numbers are consistent with those observed in the starbursting region of M82, where $\lambda \approx 2-7 \mathrm{pc}$ (Lord et al. 1996; Förster Schreiber et al. 2001) and [C II]/FIR $\approx(1-3) \times 10^{-3}$ (this work and Contursi et al. 2013). Regarding the efficiency of the photoelectric heating, as $\Sigma_{\text {FIR }}$ increases from $\Sigma_{\text {FIR }} \sim 10^{9}$ to $10^{12} L_{\odot} \mathrm{kpc}^{-2}$, the charging parameter increases from $\gamma \sim 1 \times$ $10^{3}$ to $\sim 8 \times 10^{6} \mathrm{~K}^{1 / 2} \mathrm{~cm}^{-3}$. ${ }^{12}$ This nearly four orders of magnitude increment in the charging parameter implies a reduction in the photoelectric heating efficiency, and hence the [C II]/FIR ratio, of a factor of $\sim 500$ due to the charging of the dust grains. The net result is that, for a fixed neutral cloud density, the [C II]-to-FIR ratio decreases as $G_{0} / n_{\mathrm{H}}$, and consequently $\Sigma_{\mathrm{FIR}}$ increases.

The majority of the [C II]-to-FIR ratios observed in our galaxies lie between the dense PDR and average ISRF model curves. This is expected as, in reality, the ISM structure is a combination of these two scenarios. For example, Parravano et al. (2003) find that averaged over the lifetime of a massive

\footnotetext{
12 These minimum and maximum values of $\gamma$ are similar to the values found in the Horsehead nebula and the NW component of NGC 7023, respectively (Okada et al. 2013).
} 

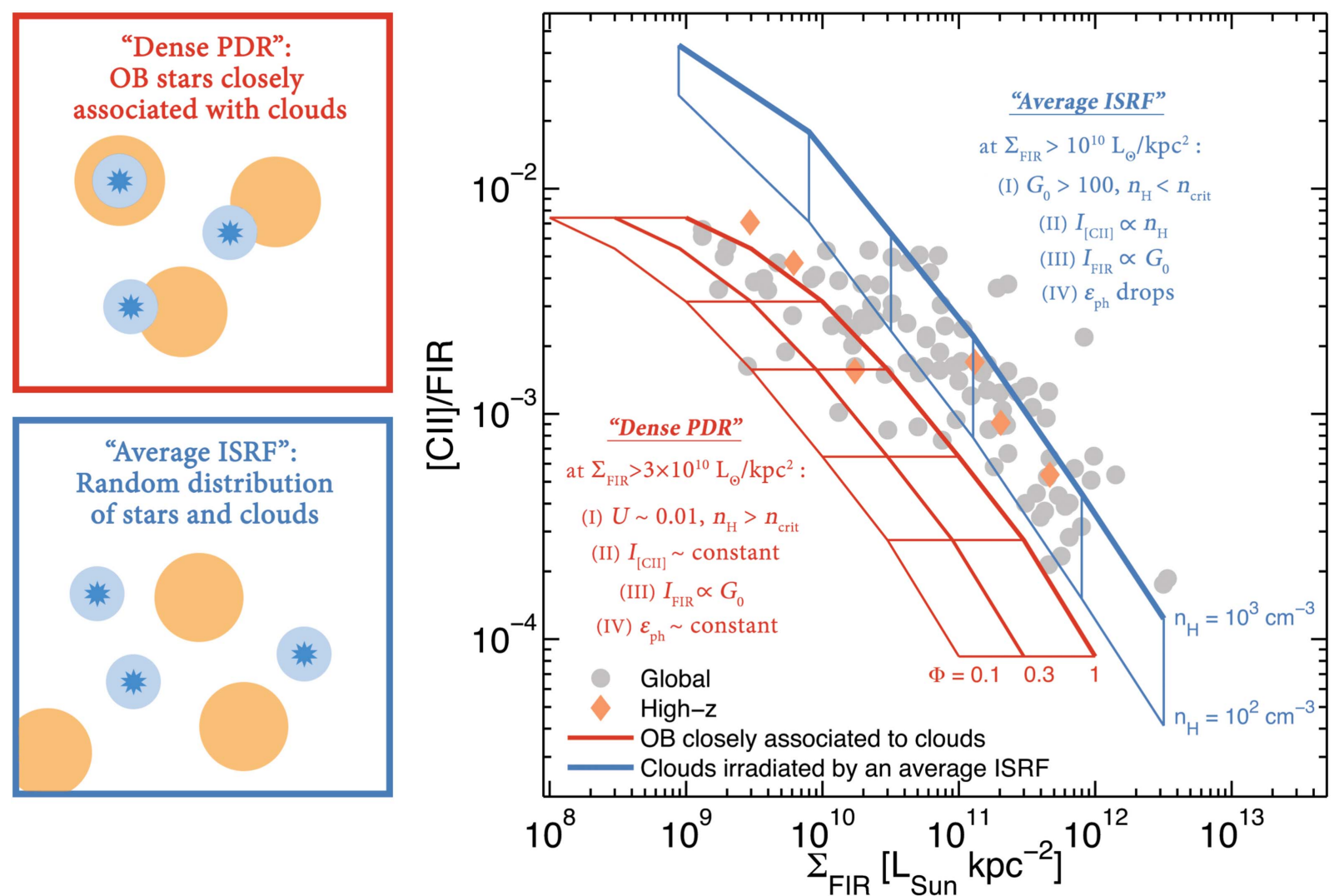

Figure 1. [C II]-to-FIR ratio as a function of $\Sigma_{\mathrm{FIR}}$ observed in nearby (gray circles) and high- $z(z>4)$ galaxies (orange diamonds). To explore the physical processes that drive the [C II] deficit, we consider a toy model defined by two extreme scenarios; one where all OB stars are closely associated with molecular gas clouds (red box), and one where OB associations and gas clouds are randomly distributed (blue box). We overplot the model results for both scenarios: (1) dense PDRs varying the beam area filling factor $\Phi$ from 0.1 to 1 (red lines), and (2) random distribution of OB stars and clouds varying the neutral gas density $n_{\mathrm{H}}$ from $10^{2}$ to $10^{3} \mathrm{~cm}^{-3}$ (blue lines).

cluster, $\sim 20 \%$ of the FUV photons produced by massive stars end up illuminating nearby dense molecular clouds, while $\sim 80 \%$ reaches the diffuse ISM. In the Orion molecular complex, Goicoechea et al. (2015) find that $\sim 85 \%$ of the total [C II] luminosity arises from a dense PDR component (with typical $\left[\mathrm{C}\right.$ II] $/$ FIR ratios in the $\sim 10^{-4}-10^{-3}$ range, $L_{\mathrm{FIR}} / M_{\mathrm{H}_{2}} \gtrsim 80 L_{\odot} M_{\odot}^{-1}, G_{0} \gtrsim 10^{4}$, and $n_{\mathrm{H}} \gtrsim 10^{5} \mathrm{~cm}^{-3}$ ) and an extended cloud component (with [C II]/FIR ratios in the $\sim 10^{-3}-10^{-2}$ range)

The threshold value of $\Sigma_{\mathrm{FIR}} \sim 3 \times 10^{10} L_{\odot} \mathrm{kpc}^{-2}$ at which our toy model predicts a decrease in the [C II]/FIR ratio is remarkably similar to the $\Sigma_{\mathrm{FIR}}$ value that separates galaxies with normal modes of star formation from compact starbursts (Elbaz et al. 2011), and PDR regimes having constant or increasing $G_{0} / n_{\mathrm{H}}$ ratios as a function of $\Sigma_{\mathrm{FIR}}$ (Díaz-Santos et al. 2017). In addition, SHINING galaxies with $\Sigma_{\text {FIR }} \gtrsim 3 \times 10^{10} L_{\odot} \mathrm{kpc}^{-2}$ tend to have $L_{\mathrm{FIR}} / M_{\mathrm{mol}} \gtrsim 50 L_{\odot} M_{\odot}^{-1}$, which is characteristic of galaxies with a faster and/or more efficient mode of star formation (e.g., Daddi et al. 2010; Genzel et al. 2010; GraciáCarpio et al. 2011). This suggests that the mean properties of PDRs and $\mathrm{HII}$ regions in these two groups of galaxies are different. Galaxies with $\Sigma_{\text {FIR }} \gtrsim 3 \times 10^{10} L_{\odot} \mathrm{kpc}^{-2}$ have more compact, dense PDR/H II region complexes powered by a higher fraction of young, massive stars that produce harsh radiation fields in their vicinity, and a higher average radiation field in the disk. All of these combined produce global [C II]/FIR ratios that are lower compared to those in galaxies with normal and more extended star formation activity.
As a cautionary note, it is important to mention that additional physical effects not considered in our models can also contribute to the [C II] deficit, including [C II] selfabsorption and high dust opacity in very obscured, dense starbursts (e.g., Arp 220; González-Alfonso et al. 2004; Rangwala et al. 2011; Scoville et al. 2017), or the impact of a very powerful AGN on the ionization state of the gas (e.g., Langer \& Pineda 2015). Regarding other models and simulations that address the problem of the [C II] deficit, we refer to Abel et al. (2009), Fischer et al. (2014), GonzálezAlfonso et al. (2008), González-Alfonso et al. (2015), Muñoz \& Oh (2015), Langer \& Pineda (2015), Narayanan \& Krumholz (2017), and Díaz-Santos et al. (2017). In particular, González-Alfonso et al. (2015) use a composite model that simultaneously describes the $\mathrm{OH}$ absorption, the high $L_{\mathrm{FIR}} / M_{\text {mol }}$ ratios, and the [C II] deficit observed in local (U) LIRGs. The model consists of a warm, optically thick component that is responsible for the molecular-absorptiondominated spectra in (U)LIRGs and that emits inefficiently in [C II] emission, and a colder, optically thin component with a fix density of $n_{\mathrm{H}}=10^{3} \mathrm{~cm}^{-3}$ that produces the bulk of the [C II] emission. In this model, the [C II] deficit—-that is correlated with the equivalent width of the absorbing $\mathrm{OH}$ $65 \mu \mathrm{m}$ line and $L_{\mathrm{FIR}} / M_{\mathrm{mol}}$-is a consequence of the limited reservoir of $\mathrm{C}^{+}$ions that at high luminosity-to-gas mass ratios limits the [C II] luminosity per unit of luminous power in the FIR continuum (see, for example, Equation (5) in GonzálezAlfonso et al. 2008). 

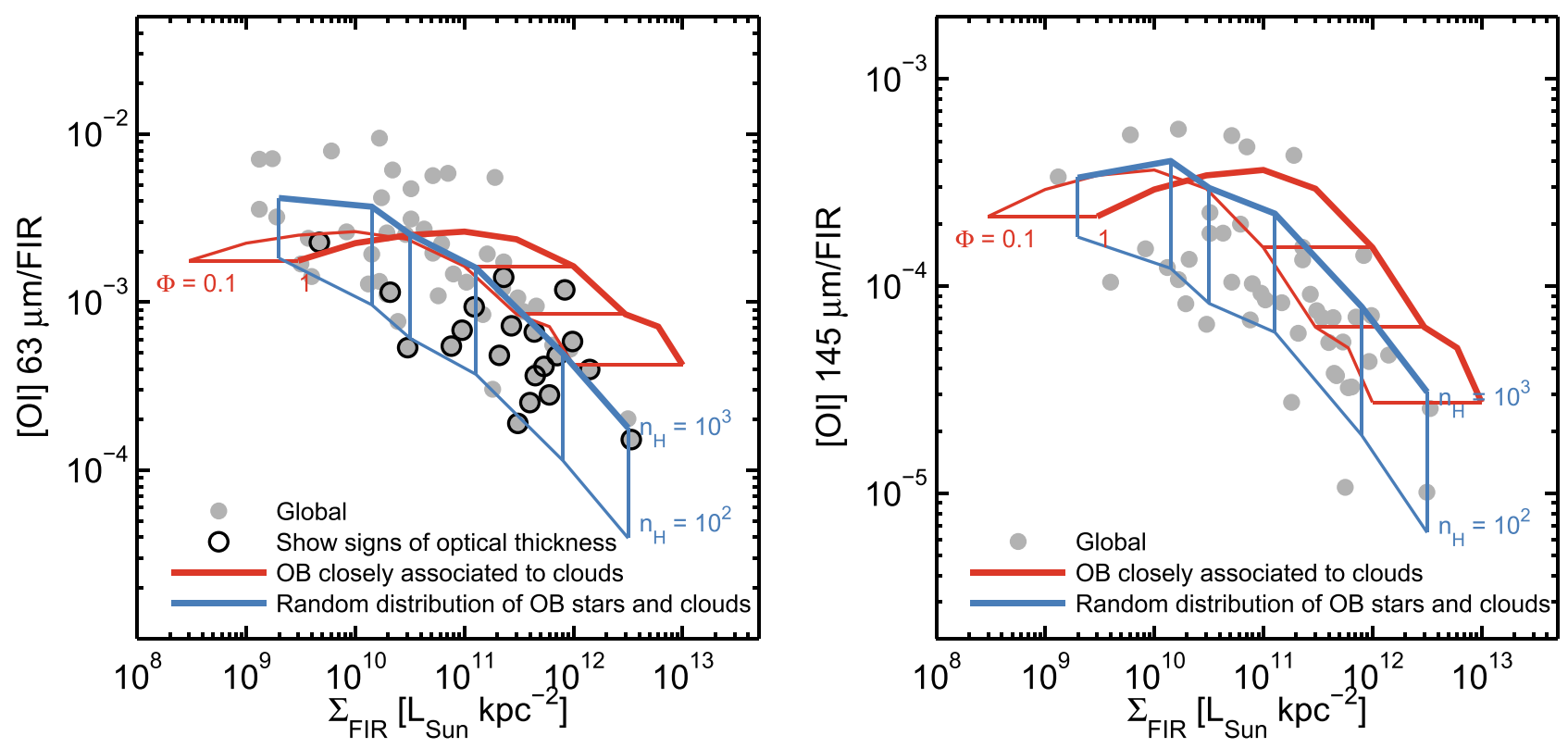

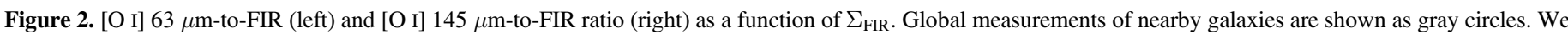
overplot the model results for both scenarios: (1) dense PDRs varying the beam area filling factor $\Phi$ from 0.1 to 1 (red lines), and (2) random distribution of OB stars and clouds varying the neutral gas density $n_{\mathrm{H}}$ from $10^{2}$ to $10^{3} \mathrm{~cm}^{-3}$ (blue lines). Galaxies that show some degree of optical thickness in their [O I] $63 \mu \mathrm{m}$ line emission (identified as sources with [O I] 63/145 $\mu \mathrm{m}$ line ratios $<10$, and assuming that the [O I] $145 \mu \mathrm{m}$ line emission is optically thin) are marked with a black border.

Figure 2 shows the comparison between our models and the observed [O I]-to-FIR ratios as a function of $\Sigma_{\text {FIR. In the case of }}$ [O I] $63 \mu \mathrm{m}$ (left panel), both models overlap and agree reasonably well with the observations until reaching $\Sigma_{\mathrm{FIR}} \approx 3 \times 10^{10} L_{\odot} \mathrm{kpc}^{-2}$. Above this surface brightness, the model predictions differ: for clouds irradiated by an average ISRF, $\left\langle G_{0}\right\rangle^{\text {ISRF }}$ reaches $\sim 400$, which results in the high-temperature saturation of the [O I] $63 \mu \mathrm{m}$ line and the decline of the [O I] $63 \mu \mathrm{m}$-to-FIR ratio with $\Sigma_{\mathrm{FIR}}$. On the other hand, in the dense PDR formulation, we do not predict a decline in the [O I] $63 \mu \mathrm{m}$-to-FIR ratio until we reach IR surface brightnesses of the order of $\Sigma_{\mathrm{FIR}} \approx 5 \times 10^{11} L_{\odot} \mathrm{kpc}^{-2}$. This threshold $\Sigma_{\text {FIR }}$ value is significantly higher than in the [C II] case due to the fact that the critical density of the [O I] $63 \mu \mathrm{m}$ line $\left(n_{\text {crit }}=4.7 \times 10^{5} \mathrm{~cm}^{-3}\right)$ is a factor $\times 150$ higher than the critical density of the [C II] line.

At first sight, it seems that the decline in the observed [O I] $63 \mu \mathrm{m}$-to-FIR ratio is better described by the scenario of clouds irradiated by an average ISRF. However, when interpreting [O I] $63 \mu \mathrm{m}$ observations, it is important to consider that this line can become optically thick (e.g., Poglitsch et al. 1996; Malhotra et al. 2001; Farrah et al. 2013; Rosenberg et al. 2015) and suppressed through selfabsorption and dust extinction (e.g., Luhman et al. 2003; González-Alfonso et al. 2004, 2008, 2012; Vasta et al. 2010). In general, galaxies with [O I] $63 / 145 \mu \mathrm{m}$ ratios lower than $\sim 10$ (assuming that [O I] $145 \mu \mathrm{m}$ is optically thin) can be considered to exhibit some degree of optical thickness in the [O I] $63 \mu \mathrm{m}$ emission. In Figure 2, we mark galaxies in this category using thick black circles. These systems correspond to the brightest galaxies in our sample, and we cannot discard that their unabsorbed [O I] $63 \mu \mathrm{m}$-to-FIR ratios are higher and in better agreement with the dense PDR model results.

Finally, the right panel of Figure 2 shows the results for the [O I] $145 \mu \mathrm{m}$ line. Overall there is a good agreement between the model results and the observed [O I] $145 \mu$ m-to-FIR ratios.
The characteristics of the model outputs are also similar to that of the [O I] $63 \mu \mathrm{m}$ line, except that in the dense PDR scenario the [O I] $145 \mu$ m-to-FIR ratio stars to drop at a lower $\Sigma_{\text {FIR value. The }}$ reason is that the critical density of the [O I] $145 \mu \mathrm{m}$ transition is a factor of $\sim 5$ lower than the critical density of the [O I] $63 \mu \mathrm{m}$ line.

\subsection{Cloudy Modeling of the Observed Infrared Line Ratio Trends}

In addition to the analysis of the line-to-continuum trends based on our toy model, in this section we continue with the interpretation of the observed line ratios using the framework built by Abel et al. (2009) and Fischer et al. (2014) based on the Cloudy spectral synthesis code (Ferland et al. 1999, 2013).

The models (described in detail in Fischer et al. 2014) consider a spherical one-dimensional geometry, where the central source of heating is dominated by a young starburst or an AGN. Cloudy is used to compute the thermal and chemical structure of the gas cloud from the illuminated surface of hot, ionized hydrogen into regions with high hydrogen column density $\left(N_{\mathrm{H}}\right)$ and optical extinction $\left(A_{\mathrm{V}}\right)$, where atoms have combined into molecules. The ionized gas density of the illuminated face of the cloud was set by Fischer et al. (2014) to $n_{\mathrm{H}^{+}}=30,300$, or $3000 \mathrm{~cm}^{-3}$, and calculations of the line intensities and dust continuum are measured as a function of the ionization parameter $(U)$ up to hydrogen column densities of $N_{\mathrm{H}}=10^{25} \mathrm{~cm}^{-2}\left(A_{\mathrm{V}}=4000 \mathrm{mag}\right)$. The advantage of using Cloudy is that the code incorporates in one model several processes relevant to the modeling of the ISM conditions, including photoionization and photodissociation, cosmic-ray ionization and heating, photoelectric heating of gas as a function of dust grain properties, and the effect of thermal, radiation, and magnetic pressure. On the other hand, one limitation of the simple geometry assumed by the model is that emission from extra-nuclear regions is not included, which can be relevant for galaxies where star formation is distributed throughout the disk, rather than at a single central position. 
To facilitate the comparison between the observed line ratios and the model predictions, we divide our galaxies into three groups based on their FIR surface brightness $\left(\Sigma_{\mathrm{FIR}} \geqslant 10^{12}\right.$ $L_{\odot} \mathrm{kpc}^{-2}, \quad 10^{11} \leqslant \Sigma_{\mathrm{FIR}}<10^{12} L_{\odot} \mathrm{kpc}^{-2}, \quad$ and $\quad \Sigma_{\mathrm{FIR}}<10^{11}$ $L_{\odot} \mathrm{kpc}^{-2}$ ). These three categories represent groups of galaxies with line deficits that range from strong to moderate. For each group, we measure the mean ([C II $]+[\mathrm{O}$ I $] 63 \mu \mathrm{m}+[\mathrm{O} \mathrm{I}] 145 \mu \mathrm{m}) /$ FIR, [N II] $122 \mu \mathrm{m} / \mathrm{FIR}$, and [N III] $57 \mu \mathrm{m} /[\mathrm{N}$ II] $122 \mu \mathrm{m}$ ratios. These are proxies for the cooling budget and the gas heating efficiency, the ionizing photon flux, and the hardness of the UV radiation field, respectively. We find that as the FIR surface brightness of galaxies increases from $\Sigma_{\mathrm{FIR}} \sim 10^{10}$ to $\sim 10^{12} L_{\odot} \mathrm{kpc}^{-2}$, the mean ([C II] $+[\mathrm{O}$ I $\left.] 63 \mu \mathrm{m}+[\mathrm{O} \mathrm{I}] 145 \mu \mathrm{m}\right) /$ FIR, [N II] $122 \mu \mathrm{m} / \mathrm{FIR}$, and [N III] $57 \mu \mathrm{m} /[\mathrm{N}$ II] $122 \mu \mathrm{m}$ ratios drop by factors of $\sim 10, \sim 4$, and $\sim 2$, respectively.

Figure 3 shows the model contours representing the mean ratios for the three galaxy categories as a function of $U$ and $N_{\mathrm{H}}$. We assume an electron density for the illuminated face of $\mathrm{H}^{+}$gas of $n_{\mathrm{H}^{+}}=300 \mathrm{~cm}^{-3}$, in agreement with observed electron densities in central kiloparsec size regions with $\Sigma_{\mathrm{FIR}} \sim$ $10^{10-11} L_{\odot} \mathrm{kpc}^{-2}$ (Herrera-Camus et al. 2016). The left and right panels show the model predictions when assuming a starburst or AGN central source, respectively. In both cases the observed drop in the $([\mathrm{C} \mathrm{II}]+[\mathrm{O} \mathrm{I}] 63 \mu \mathrm{m}+[\mathrm{O} \mathrm{I}] 145 \mu \mathrm{m}) / \mathrm{FIR}$ and [N II] $122 \mu \mathrm{m} /$ FIR ratios can be explained by increasing both $N_{\mathrm{H}}$ and $U$. The [N III] $57 \mu \mathrm{m} /[\mathrm{N}$ II] $122 \mu \mathrm{m}$ ratio, on the other hand, is not very sensitive to changes in the ionization parameter, and its orthogonality with respect to the other line-to-continuum ratios can be used to constrain the characteristic values of $N_{\mathrm{H}}$ and $U$ for each galaxy group. In the figure, we mark with circles (squares) the position in the $U, N_{\mathrm{H}}$ plane where the ([C II] $+[\mathrm{O}$ I] $63 \mu \mathrm{m}+[\mathrm{O}$ I] $145 \mu \mathrm{m}) /$ FIR ([N II] $122 \mu \mathrm{m} / \mathrm{FIR})$ and the [N III] $57 \mu \mathrm{m} /[\mathrm{N}$ II] $122 \mu \mathrm{m}$ contours intersect.

In the starburst dominated scenario, the intersection of the contours for the $\Sigma_{\mathrm{FIR}} \sim 10^{10} L_{\odot} \mathrm{kpc}^{-2}$ galaxies falls outside the probed parameter space, but the trends seems to indicate that the contours will overlap around $N_{\mathrm{H}} \sim 10^{23.5} \mathrm{~cm}^{-2}$ and $U \sim 10^{-4.2}$. For galaxies with $\Sigma_{\mathrm{FIR}} \sim 10^{11} L_{\odot} \mathrm{kpc}^{-2}$, we find characteristic values of $N_{\mathrm{H}} \sim 10^{23.8} \mathrm{~cm}^{-2}$ and $U \sim 10^{-3.5}$, and for the $\Sigma_{\text {FIR }} \sim 10^{12} L_{\odot} \mathrm{kpc}^{-2}$ group, we find $N_{\mathrm{H}} \sim 10^{24.1} \mathrm{~cm}^{-2}$ and $U \sim 10^{-2.7}$. For an AGN central SED, we observe that the decrease in the relative intensity of the PDR and [N II] $122 \mu \mathrm{m}$ lines of galaxies in the $\Sigma_{\mathrm{FIR}} \sim 10^{10-12} L_{\odot} \mathrm{kpc}^{-2}$ range can be explained by increasing $N_{\mathrm{H}}$ from $\sim 10^{23.5}$ to $\sim 10^{24} \mathrm{~cm}^{-2}$ and $U$ from $\sim 10^{-3}$ to $\sim 10^{-2}$.

In conclusion, we find that regardless of the choice of central power source, the models can reproduce the PDR and [N II] $122 \mu \mathrm{m}$ line deficits observed in galaxies in the $\Sigma_{\mathrm{FIR}} \sim 10^{10-12} L_{\odot} \mathrm{kpc}^{-2}$ range by increasing both $N_{\mathrm{H}}$ and $U$ by factors of $\sim 4$ and $\sim 10$, respectively. This is consistent with the results found by Graciá-Carpio et al. (2011). The high column densities $\left(N_{\mathrm{H}} \sim 10^{24} \mathrm{~cm}^{-2}\right)$ predicted for the $\Sigma_{\mathrm{FIR}} \sim$ $10^{12} L_{\odot} \mathrm{kpc}^{-2}$ galaxies are compatible with the low mid-IR line to FIR continuum ratios (Farrah et al. 2013) and high $\mathrm{OH}$ and $\mathrm{H}_{2} \mathrm{O}$ column densities (e.g., González-Alfonso et al. 2012, 2015 ) observed in these systems. In addition, the high- $U$ values are required to maintain the observed warm FIR colors (see also Fischer et al. 2014). This Cloudy model interpretation of our results is also consistent with our toy model calculations. In both cases, we find that strong-line deficits are associated with gas clouds with $U \sim 10^{-2}$. At this high $U$ value, the absorption of UV photons by dust in the ionized region is significant, plus the density of the gas can exceed the critical density of the [C II] line (e.g., our toy model results or Figure 1 in Abel et al. 2009).

\subsection{The Influence of AGN Activity on the [C II]/FIR Ratio}

X-ray dominated regions (XDRs) produced by AGNs heat the surrounding gas and dust, and this can have a significant impact on the (local) [C II]/FIR ratio (e.g., Maloney et al. 1996; Meijerink \& Spaans 2005). In particular, harsh radiation fields present in XDRs can decrease the cooling power of [C II] by (1) destroying small dust grains and PAHs, thus reducing the photoelectric heating efficiency of the gas (Voit 1992); (2) converting a fraction of the $\mathrm{C}^{+}$ions to higher ionization states (Langer \& Pineda 2015); and (3) heating the dust and gas to temperatures high enough for the [O I] $63 \mu \mathrm{m}$ line to become the dominant cooling channel (e.g., Abel et al. 2009).

To explore the connection between the AGN power and its influence on the dust continuum and PDR line emission, in Figure 4, we plot the [C II]/FIR (left) and [O I] $63 \mu \mathrm{m} / \mathrm{FIR}$ (right) ratio as a function of the $\mathrm{X}$-ray luminosity $\left(L_{\mathrm{X} \text {-ray }}\right)$ for ULIRGS, $z \sim 1$ galaxies from Stacey et al. (2010; 3 AGN dominated, 1 mixed, and 2 starburst dominated systems), and resolved regions in Seyfert SHINING galaxies. The absorptioncorrected X-ray luminosities in the $2-10 \mathrm{keV}$ range were retrieved from Iyomoto et al. (1997), Cappi et al. (2006), Teng \& Veilleux (2010), and de Rosa et al. (2012). The gray solid line shows the model predictions from Langer \& Pineda (2015) for the $[\mathrm{C} \mathrm{II}] /$ FIR ratio as a function of X-ray luminosity. ${ }^{13} \mathrm{In}$ this model, the [C II] emission arises from dense warm ionized gas and PDRs, and the main effect of the presence of a strong source of X-ray flux is to convert a fraction of the $\mathrm{C}^{+}$ions to higher ionization states, thus reducing the [C II] luminosity. They predict this effect should reduce the [C II]/FIR ratio by a factor of a few at $L_{\mathrm{X} \text {-ray }} \approx 10^{44} \mathrm{erg} \mathrm{s}^{-1}$, and by an order of magnitude at $L_{\mathrm{X} \text {-ray }} \gtrsim 10^{45} \mathrm{erg} \mathrm{s}^{-1}$.

To study the local effect of the AGN on the [C II]/FIR ratio, we use the SHINING Seyfert galaxies that are spatially resolved to compare the $[\mathrm{C} \mathrm{II}] /$ FIR ratio measured in the central spaxel versus the median of the disk (excluding the central spaxel). For the four galaxies with $L_{\mathrm{X} \text {-ray }} \lesssim 10^{42} \mathrm{erg} \mathrm{s}^{-1} \quad$ (NGC 1365, NGC 5033, Centaurus A, and Circinus), we observe a central [C II]/FIR ratio a factor of 2-3 lower compared to the disk's median. Interestingly, the central regions in these systems do not show an $[\mathrm{OI}] 63 \mu \mathrm{m} / \mathrm{FIR}$ ratio deficit relative to the disk, in fact, NGC 5033 and Centaurus A have central [O I] $63 \mu \mathrm{m} / \mathrm{FIR}$ ratios a factor of $\sim 2$ higher than the median of their disks. This suggests that the suppression of the central [C II]/FIR ratio in these Seyfert galaxies is a consequence of the dominant contribution of the [O I] $63 \mu \mathrm{m}$ line to the cooling of the neutral gas.

The remaining SHINING Seyfert galaxy that is spatially resolved is the archetypical system NGC 1068. The [C II]/FIR ratio measured in the central spaxel (spatial scale $\sim 500 \mathrm{pc}$ ) is $6 \times 10^{-4}$, a value that is typical of those observed in ULIRGs, and a factor of $\sim 6$ lower than the median ratio measured in its disk. Similar to the other Seyfert galaxies in our sample, we do not observe central suppression of the [O I] $63 \mu \mathrm{m} / \mathrm{FIR}$ ratio relative to the disk. As a consequence, the cooling of the

\footnotetext{
13 The X-ray luminosities computed in the Langer \& Pineda (2015) model are integrated for energies larger than $1 \mathrm{keV}$, while the X-ray luminosities of the galaxies included in Figure 4 cover the $2-10 \mathrm{keV}$ range. As described by Langer \& Pineda (2015), if we assume an X-ray photon index of $\Gamma=1.9$ this implies that data points should shift $\sim 0.6$ dex to the right.
} 

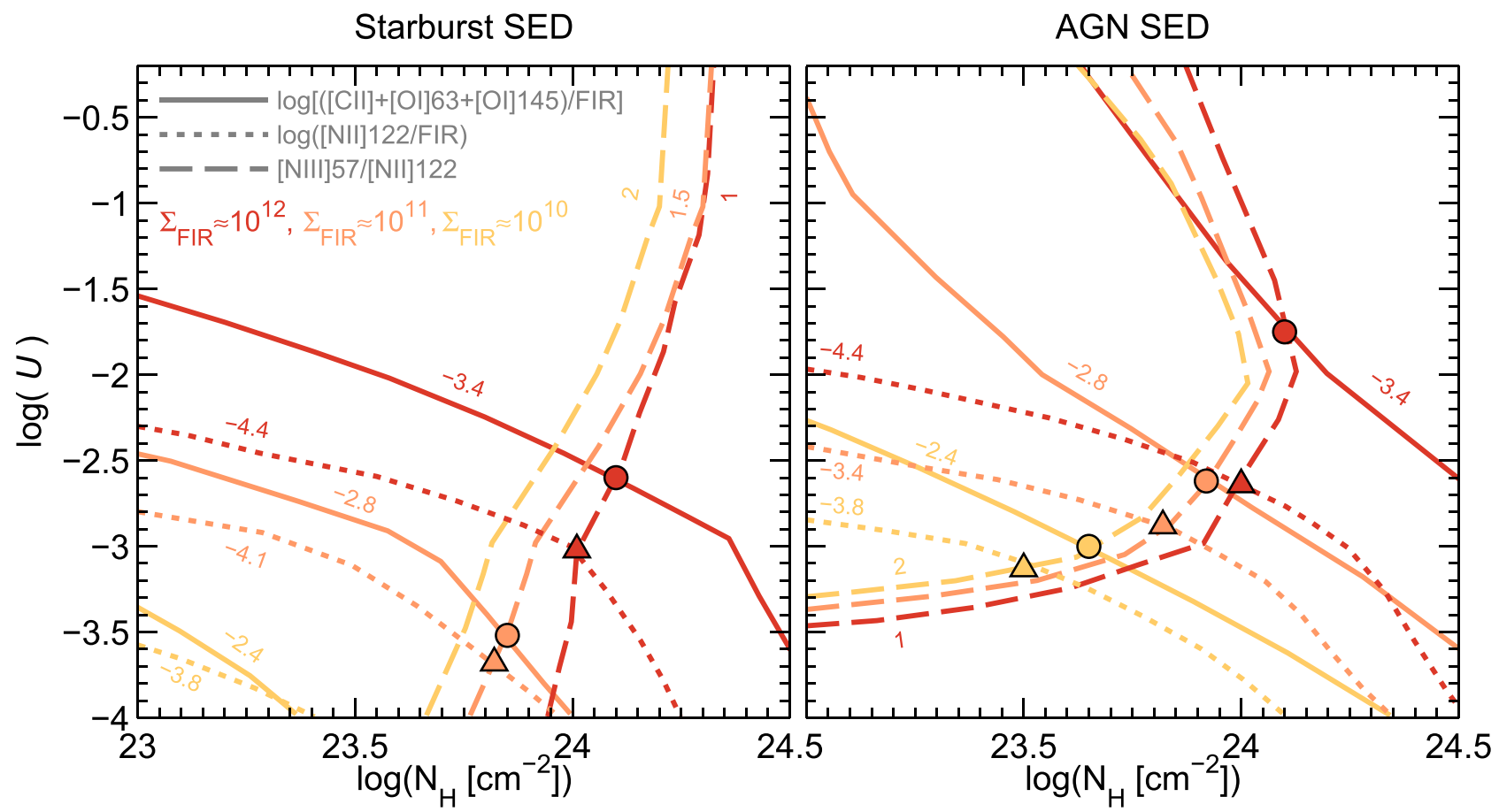

Figure 3. Cloudy predictions for the ([C II] $+[\mathrm{O}$ I] $63 \mu \mathrm{m}+[\mathrm{O}$ I] $145 \mu \mathrm{m}$ )/FIR (solid contours), [N II] $122 \mu \mathrm{m} / \mathrm{FIR}$ (dotted contours), and [N III] $57 \mu \mathrm{m} /[\mathrm{N}$ II] $122 \mu \mathrm{m}$ (dashed contours) ratios as a function of hydrogen column density $\left(N_{\mathrm{H}}\right)$ and ionization parameter $(U)$ assuming starburst (left) or AGN (right) central illumination SEDs (Fischer et al. 2014). The contour values are chosen to represent the mean values measured in galaxies with $\Sigma_{\mathrm{FIR}} \approx 10^{10} L_{\odot} \mathrm{kpc}^{-2}$ (yellow), $\Sigma_{\mathrm{FIR}} \approx 10^{11} L_{\odot} \mathrm{kpc}^{-2}$ (orange), and $\Sigma_{\mathrm{FIR}} \approx 10^{12} L_{\odot} \mathrm{kpc}^{-2}$ (red). For each galaxy group, we mark with a circle the intersection between the ([C II] $+[\mathrm{O}$ I] $63 \mu \mathrm{m}$ $+[\mathrm{O}$ I] $145 \mu \mathrm{m}$ )/FIR and [N III] $57 \mu \mathrm{m} /[\mathrm{N}$ II] $122 \mu \mathrm{m}$ curves, and with a triangle the intersection between the [N II] $122 \mu \mathrm{m} / \mathrm{FIR}$ and [N III] $57 \mu \mathrm{m} /[\mathrm{N}$ II] $122 \mu \mathrm{m}$ curves. The overlapping regions of the model results shows that the observed line deficit of the PDR lines can be explained as a result of the increase in the hydrogen column density and the ionization parameter.

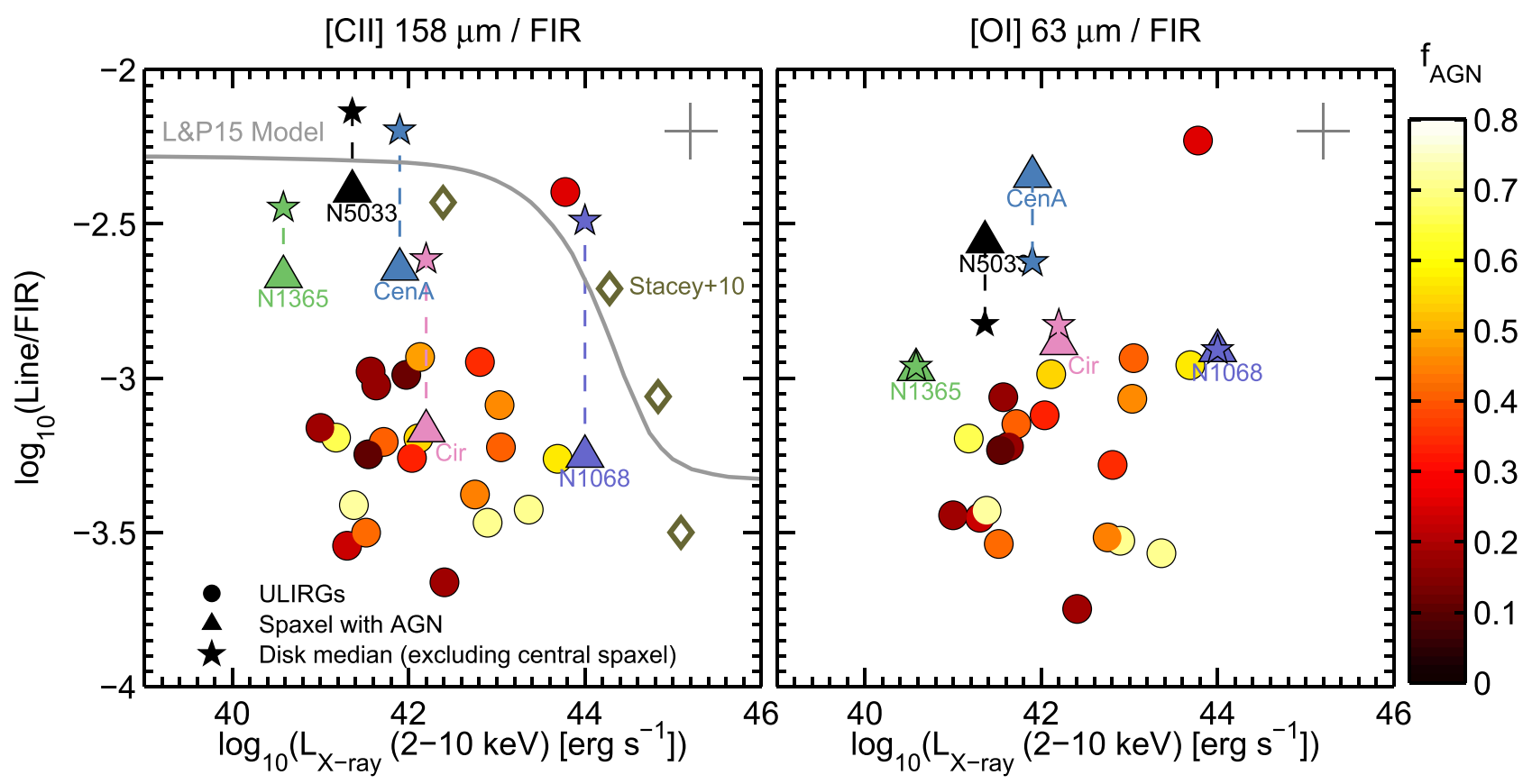

Figure 4. (Left) $[\mathrm{C} \mathrm{II}]$ /FIR ratio as a function of intrinsic (absorption-corrected) X-ray luminosity (2-10 keV) of ULIRGs (color circles), the central spaxel (triangles) and the median of the disk excluding the central spaxel (stars) of five resolved SHINING AGN galaxies, and galaxies in the Stacey et al. (2010) sample (empty dark green diamonds). For the ULIRGS, the color scale indicates the fractional contribution of nuclear activity to the bolometric luminosity $\left(f_{\mathrm{AGN}}\right)$ as calculated by Veilleux et al. (2009b). The gray solid line represents the model predictions by Langer \& Pineda (2015) assuming a PDR filling factor of 0.002. (Right) Similar to the left panel, but this time the ordinate indicates the [O I] $63 \mu \mathrm{m} / \mathrm{FIR}$ ratio. A typical error bar is plotted in the upper-right corner.

neutral gas in the central spaxel of NGC 1068 is dominated by the [O I] $63 \mu \mathrm{m}$ line (the [O I] $63 \mu \mathrm{m} /[\mathrm{C}$ II] line luminosity ratio is 2.3). The strong central X-ray source in NGC 1068 place this galaxy in the regime where the Langer \& Pineda (2015) model predicts that changes in the overall state ionization of the gas can contribute to the $[\mathrm{CII}] / \mathrm{FIR}$ suppression. Additional 
evidence that supports this scenario is that the ratio between the [O III] $88 \mu \mathrm{m}$ line (which originated in highly ionized gas) and the [C II] line in the central spaxel is [O III] $88 \mu \mathrm{m} /[\mathrm{C} \mathrm{II}]=1.3$, one of the highest values in the entire sample of resolved and unresolved SHINING galaxies (see Figure 11 in Paper I; Herrera-Camus et al. 2018).

For the ULIRGs in our sample, we only have global measurements, which implies that any impact of the AGN on the $[\mathrm{C} \mathrm{II}] /$ FIR ratio will be diluted by the emission from the star-forming disk. One alternative, however, to assess the influence of the AGN is to consider in the analysis what fraction of the bolometric luminosity is contributed by the nuclear activity. This fraction is found to be, on average, $\sim 30 \%$ in $\mathrm{H}$ II-like and LINERs ULIRGS and $\sim 50 \%-75 \%$ in warm Seyfert ULIRGs (Veilleux et al. 2009b). We observe that all the ULIRGs in our sample are characterized by very low [C II]/ FIR ratios $\left(5 \times 10^{-4} \lesssim[\mathrm{C}\right.$ II $\left.] / F I R \lesssim 2 \times 10^{-3}\right)$, and this seems to be independent of how much of the bolometric luminosity is dominated by the AGN, or how powerful the AGN is in terms of X-ray luminosity. The Kendall $\tau$ correlation coefficient of [C II]/FIR versus AGN luminosity and AGN fraction are $\tau=0.06(p=0.69)$ and $\tau=-0.18(p=0.23)$, respectively. In fact, ULIRGs show [C II]/FIR ratios as low as those predicted in the most extreme cases of the Langer \& Pineda (2015) model, and comparable to those observed in the $z \sim 1$ AGN systems that have X-ray luminosities $L_{\mathrm{X} \text {-ray }} \gtrsim$ $10^{45} \mathrm{erg} \mathrm{s}^{-1}$ (Stacey et al. 2010). The situation is similar for the [O I] $63 \mu \mathrm{m}$ line, as we do not observe a trend of increasing (or decreasing) [O I] $63 \mu \mathrm{m} / \mathrm{FIR}$ ratios as a function of X-ray luminosity (Kendall $\tau=0.25$ with $p=0.13$ ) or AGN fraction (Kendall $\tau=-0.08$ with $p=0.63$ ).

In summary, we observe evidence for central suppression of the $[\mathrm{C} \mathrm{II}] /$ FIR ratio in the inner $\sim 500 \mathrm{pc}$ of Seyfert galaxies. However, the magnitude of this drop is similar to that observed in central regions of some star-forming galaxies, or galaxies that host weak AGNs (this work; Parkin et al. 2013; HerreraCamus et al. 2015; Smith et al. 2017). This could be explained by the different physical conditions that dominate the nucleus and the disk regions, including for the former warmer dust temperatures, the prevalence of [O I] $63 \mu \mathrm{m}$ as the main cooling channel, and in some cases additional contribution to the FIR emission by populations of old stars (e.g., Smith et al. 2017). Perhaps the only Seyfert galaxy in our sample where we find evidence for the central suppression of the $[\mathrm{C} \mathrm{II}] /$ FIR ratio as a direct consequence of the AGN X-ray emission is NGC 1068. Finally, in our sample of ULIRGs, we do not observe any correlation between the global $[\mathrm{C} \mathrm{II}] / \mathrm{FIR}$ ratio and the X-ray luminosity, or the fractional contribution of the nuclear activity to the bolometric luminosity. Thus we do not find that AGN activity plays a major role in the observed (global) [C II] deficit of ULIRGs with $L_{\mathrm{X} \text {-ray }} \lesssim 10^{44} \mathrm{erg} \mathrm{s}^{-1}$.

\section{4. [C II]-SFR Scaling Relations for Different Galaxy Types and Physical Conditions}

In thermal balance, the cooling of the neutral atomic gas dominated by the [C II] transition, traces the amount of heating by the star formation activity via the photoelectric effect on small dust grains (Hollenbach \& Tielens 1999). This, combined with the high brightness of the [C II] line, motivates the use of [C II] as a star formation tracer (e.g., Boselli et al. 2002; De Looze et al. 2014; Herrera-Camus et al. 2015). However, if we assume that the FIR emission is a reliable tracer of the star formation activity (e.g., Kennicutt \& Evans 2012), then the robustness of this tracer is limited by the large variations observed in the [C II] to FIR ratio. In order to explore the robustness of the SFR as a predictor of the [C II] luminosity (or the reliability of the $[\mathrm{C} \mathrm{II}]$ transition as a star formation tracer), in this section, we present scaling relations between [C II] emission and SFR for different types of galaxies and physical conditions.

In order to facilitate the comparison between our sample of nearby galaxies and those at high- $z$, we present our results in the context of the "main sequence" (MS) of star-forming galaxies, i.e., the \pm 0.3 dex scatter sequence relating star formation activity with galaxy stellar mass as a function of redshift (e.g., Daddi et al. 2007; Elbaz et al. 2007; Rodighiero et al. 2011; Whitaker et al. 2012). For this, we calculate the offset from the main-sequence, $\Delta \mathrm{MS}$, which corresponds to the logarithm of the specific SFR (sSFR $=\mathrm{SFR} / M_{*}$, where $M_{*}$ is the stellar mass) normalized by the stellar mass- and redshiftdependent center line of the main sequence, $\operatorname{sSFR}\left(M S, z, M_{*}\right)$. For the latter, we adopt the prescription proposed by Speagle et al. (2014):

$$
\begin{aligned}
& \log _{10}\left(\operatorname{sSFR}\left(\mathrm{MS}, z, M_{*}\right)\right)=(-0.16-0.026 t) \\
& \quad \times\left(\log _{10}\left(M_{*}\right)-0.025\right)+(2.49+0.11 t)\left(\mathrm{Gyr}^{-1}\right),
\end{aligned}
$$

where $t$ is the cosmic time in units of Gyr, and we assume a flat $\Lambda \mathrm{CDM}$ universe with $\Omega_{m}=0.3$ and $H_{0}=70 \mathrm{~km} \mathrm{~s}^{-1} \mathrm{Mpc}^{-1}$. This prescription can be applied to galaxies in the redshift range $z=0-5$ and stellar masses in the $M_{*}=10^{9-11.8} M_{\odot}$ range.

To determine the specific SFR of the galaxies in our sample, we need stellar masses and SFRs. We calculate stellar masses using the Two Micron All Sky Survey (2MASS) $K$-band $(2.2 \mu \mathrm{m})$ photometry and the $M_{*} / L$ conversion from Lacey et al. (2008). K-band luminosities can suffer from contamination from power-law emission from AGN heating; though, we expect this contamination to be minor in Seyfert 1 and 2 sources ( $\lesssim 30 \%$ according to Mushotzky et al. 2008). Note, however, that this may not be true for (U)LIRGs in our sample with high AGN fractions (e.g., Veilleux et al. 2002, 2006, 2009a). For the systems in our sample that overlap with GOALS, we measure stellar masses that are consistent with those from Howell et al. (2010; also based on 2MASS $K$-band magnitudes) and U et al. (2012; based on SED fitting of the UV-NIR part of the spectrum).

To calculate the SFRs, we use the calibration based on the total far-infrared luminosity $\left(L_{\mathrm{TIR}}(8-1000 \mu \mathrm{m})\right)$ by Murphy et al. (2011). We convert FIR(40-122 $\mu \mathrm{m})$ luminosities into $\operatorname{TIR}(8-1000 \mu \mathrm{m})$ luminosities by scaling the former by a factor of 1.75 (see Section 5.2 in Paper I; Herrera-Camus et al. 2018). How reliable is the use of the FIR emission as an SFR tracer? For galaxies with $L_{\mathrm{TIR}} \gtrsim 10^{11} L_{\odot}$, TIR emission represents the most reliable SFR indicator, as the unobscured contribution of massive stars to the total SFR is small $(\lesssim 15 \%$; Calzetti et al. 2010). In the intermediate luminosity range between $L_{\mathrm{TIR}} \sim 10^{10}-10^{11} L_{\odot}$, galaxies become more transparent at UV and optical wavelengths, and as a consequence the infrared emission by itself is less representative of the total star formation activity. However, as pointed out by Kennicutt \& Evans (2012), in this regime the effects of partial dust attenuation and dust heating by old stars roughly compensate for each other, which implies that TIR emission still trace an important fraction of the star formation activity. Finally, in 
Table 1

Summary of $L_{[\mathrm{C} \text { II] }}-\mathrm{SFR}$ and $L_{[\mathrm{C} \text { II] }}-L_{\mathrm{FIR}}$ Scalings

\begin{tabular}{llcc}
\hline \hline Class & Criteria & $\log _{10}\left(L_{[\mathrm{C} \mathrm{m}]} / \mathrm{SFR}\right)^{\mathrm{a}}$ & $\log _{10}\left(L_{[\mathrm{C} \mathrm{II}]} / L_{\mathrm{FIR}}\right)$ \\
\hline Star-forming & BPT Classification $^{\mathrm{b}}$ & $6.93 \pm 0.32$ & $-2.62 \pm 0.33$ \\
AGN & BPT Classification $^{\mathrm{b}}$ & $6.84 \pm 0.41$ & $-2.75 \pm 0.40$ \\
LINERs & BPT Classification & \\
(U)LIRGs & $L_{\mathrm{FIR}} \geqslant 10^{11} L_{\odot}$ & $6.60 \pm 0.40$ & $-2.96 \pm 0.40$ \\
\hline Main-sequence (MS) & $1 / 3 \leqslant \Delta \mathrm{MS} \leqslant 3$ & $7.63 \pm 0.37$ & $-2.95 \pm 0.37$ \\
Above MS $\times 3-20$ & $3<\Delta \mathrm{MS} \leqslant 20$ & $6.97 \pm 0.28$ & $-2.47 \pm 0.25$ \\
MS outliers $\times 20-100$ & $20<\Delta \mathrm{MS} \leqslant 100$ & $6.50 \pm 0.34$ & $-2.61 \pm 0.28$ \\
\hline Normal SFE & $L_{\mathrm{FIR}} / M_{\mathrm{mol}} \leqslant 80 L_{\odot} / M_{\odot}$ & $7.02 \pm 0.26$ & $-3.08 \pm 0.34$ \\
High SFE & $L_{\mathrm{FIR}} / M_{\mathrm{mol}}>80 L_{\odot} / M_{\odot}$ & $6.43 \pm 0.33$ & $-2.55 \pm 0.26$ \\
\hline Normal $\Sigma_{\mathrm{FIR}}$ & $10^{8} \leqslant \Sigma_{\mathrm{FIR}} \leqslant 10^{11.2 \mathrm{~d}}$ & $7.03 \pm 0.21$ & $-3.14 \pm 0.34$ \\
High $\Sigma_{\mathrm{FIR}}$ & $10^{11.2}<\Sigma_{\mathrm{FIR}} \leqslant 10^{12}$ & $6.53 \pm 0.30$ & $-2.56 \pm 0.21$ \\
\hline
\end{tabular}

Notes.

${ }^{a}$ Mean $\pm 1 \sigma$ standard deviation. Units are $L_{\odot} /\left(M_{\odot} \mathrm{yr}^{-1}\right)$. SFRs are calculated using Murphy et al. (2011) calibration based on $L_{\mathrm{TIR}}(8-1000 \mu \mathrm{m})$, where $L_{\mathrm{TIR}}(8-1000 \mu \mathrm{m})=1.75 \times L_{\mathrm{FIR}}(42.5-122.5 \mu \mathrm{m})$.

${ }^{\mathrm{b}}$ Following the Baldwin, Phillips, and Terlevich (BPT) diagnostic diagram introduced in Baldwin et al. (1981) and Veilleux \& Osterbrock (1987).

${ }^{\mathrm{c}} \mathrm{SFE}=$ star formation efficiency.

${ }^{\mathrm{d}}$ Units are $L_{\odot} \mathrm{kpc}^{-2}$.

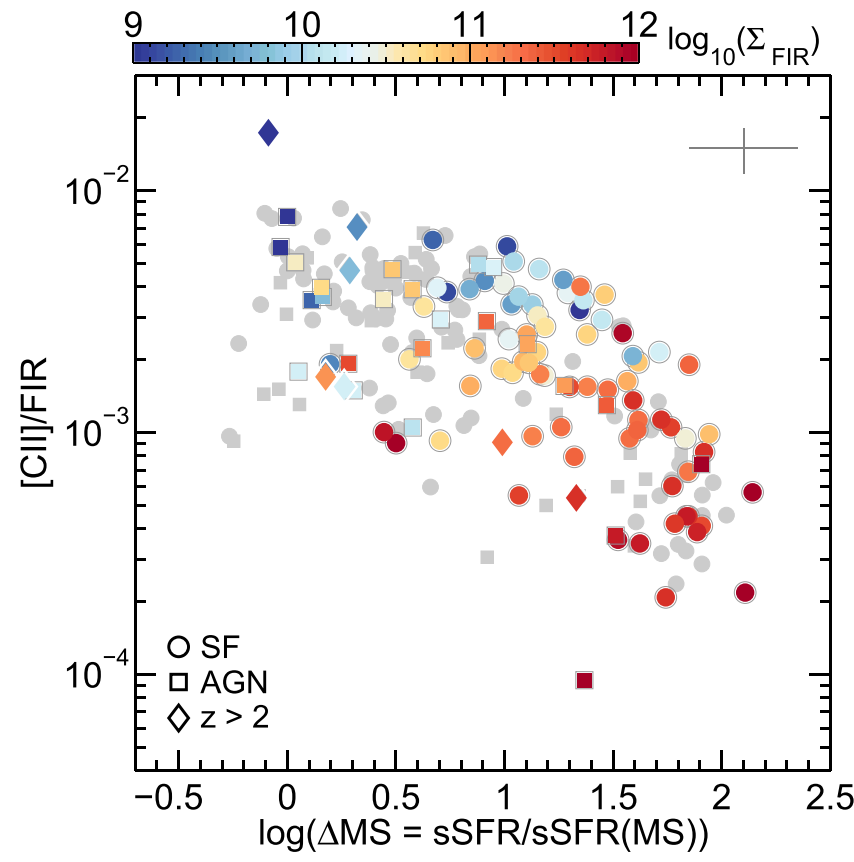

Figure 5. $[\mathrm{C} \mathrm{II}] /$ FIR ratio as a function of specific star formation rate normalized to the mid-line of the main sequence (Speagle et al. 2014) as a function of redshift $(\Delta M S)$. The color scale indicates the FIR surface brightness of the galaxies for which we have size measurements available. Starforming galaxies are shown as circles, AGNs as squares, and high- $z(z>2)$ systems galaxies as diamonds. A typical error bar is plotted in the upper-right corner.

galaxies with low dust content $\left(L_{\mathrm{TIR}} \lesssim 5 \times 10^{9} L_{\odot}\right.$; Madden et al. 2013), most of the emission produced by young, massive stars escape unabsorbed by dust, and the far-infrared emission becomes a poor tracer of the SFR (e.g.; Calzetti et al. 2010; De Looze et al. 2014). In the sample of galaxies shown in Figures 5 and $6,54 \%$ have $L_{\mathrm{TIR}}>10^{11} L_{\odot}, 41 \%$ have $L_{\mathrm{TIR}}$ in the $10^{10}-10^{11} L_{\odot}$ range, and only $5 \%$ have TIR luminosities between $6 \times 10^{9}$ and $10^{10} L_{\odot}$. In conclusion, we expect TIR emission to be a robust tracer of the star formation activity of the galaxies in our sample.

Figure 5 shows the [C II]-to-FIR ratio as a function of the offset from the main sequence of galaxies $(\Delta \mathrm{MS})$. We include local star-forming galaxies (circles), AGNs (squares), and high-z galaxies (diamonds). For the latter, we select from the literature galaxies with $L_{\mathrm{FIR}}, M_{*}$, SFR, sizes, and [C II] luminosities available. These systems are HDF850.1 $(z=5.2$; Walter et al. 2012), HFLS3 ( $z=6.34$; Riechers et al. 2013), ALESS73.1 $(z=4.8$; De Breuck et al. 2014), and four mainsequence, $z \sim 5$ galaxies from the Capak et al. (2015) sample (HZ4, 6, 9, and 10). We color code galaxies according to their FIR surface brightness (when size measurements are available). We observe that galaxies lying within a factor of $\sim 4$ of the main sequence have [C II]-to-FIR ratios in the $\sim 10^{-2}-10^{-3}$ range, while outliers above the main sequence $(\Delta \mathrm{MS} \gtrsim 10)$ have [C II]-to-FIR ratios lower than $\sim 3 \times 10^{-2}$, irrespective of whether they are classified as star-forming, AGN, or high- $z$ (see also Graciá-Carpio et al. 2011; Díaz-Santos et al. 2013; Ibar et al. 2015). This trend of decreasing [C II]-to-FIR ratio as a function of $\Delta \mathrm{MS}$ (Kendall $\tau=-0.45$ with $p<0.01$ ) is consistent with those observed as a function of $S_{63 \mu \mathrm{m}} / S_{122 \mu \mathrm{m}}$ and $L_{\mathrm{FIR}} / M_{\mathrm{mol}}$ (see Figure 4 in Herrera-Camus et al. 2018), since galaxies step along in SSFR at fixed $M_{*}$ and $z$, they have higher dust temperatures $\left(\propto S_{63 \mu \mathrm{m}} / S_{122 \mu \mathrm{m}}\right)$, star formation efficiencies $\left(\propto L_{\mathrm{FIR}} / M_{\mathrm{mol}}\right)$ (e.g., Genzel et al. 2015; Tacconi et al. 2018), and FIR surface brightnesses (e.g., Lutz et al. 2016).

In addition to the trend with the offset from the main sequence, we find that, in general, for a fixed value of $\Delta \mathrm{MS}$ the [C II]-to-FIR ratio decreases with increasing $\Sigma_{\text {FIR }}$. For example, for starburst galaxies with $\Delta \mathrm{MS} \approx 30$, those with $\Sigma_{\mathrm{FIR}} \approx 10^{10}$ $L_{\odot} \mathrm{kpc}^{-2}$ have [C II]-to-FIR ratios about an order of magnitude higher than galaxies with $\Sigma_{\mathrm{FIR}} \approx 10^{12} L_{\odot} \mathrm{kpc}^{-2}$. As we explored with our toy model (see Section 3.1), an increase in $\Sigma_{\text {FIR }}$ creates enhanced FUV radiation fields, ionization parameters, and neutral gas densities that manifest themselves in lower [C II] to FIR ratios.

We now discuss the scaling relations between [C II] luminosity and SFR that can be used to predict the [C II] 


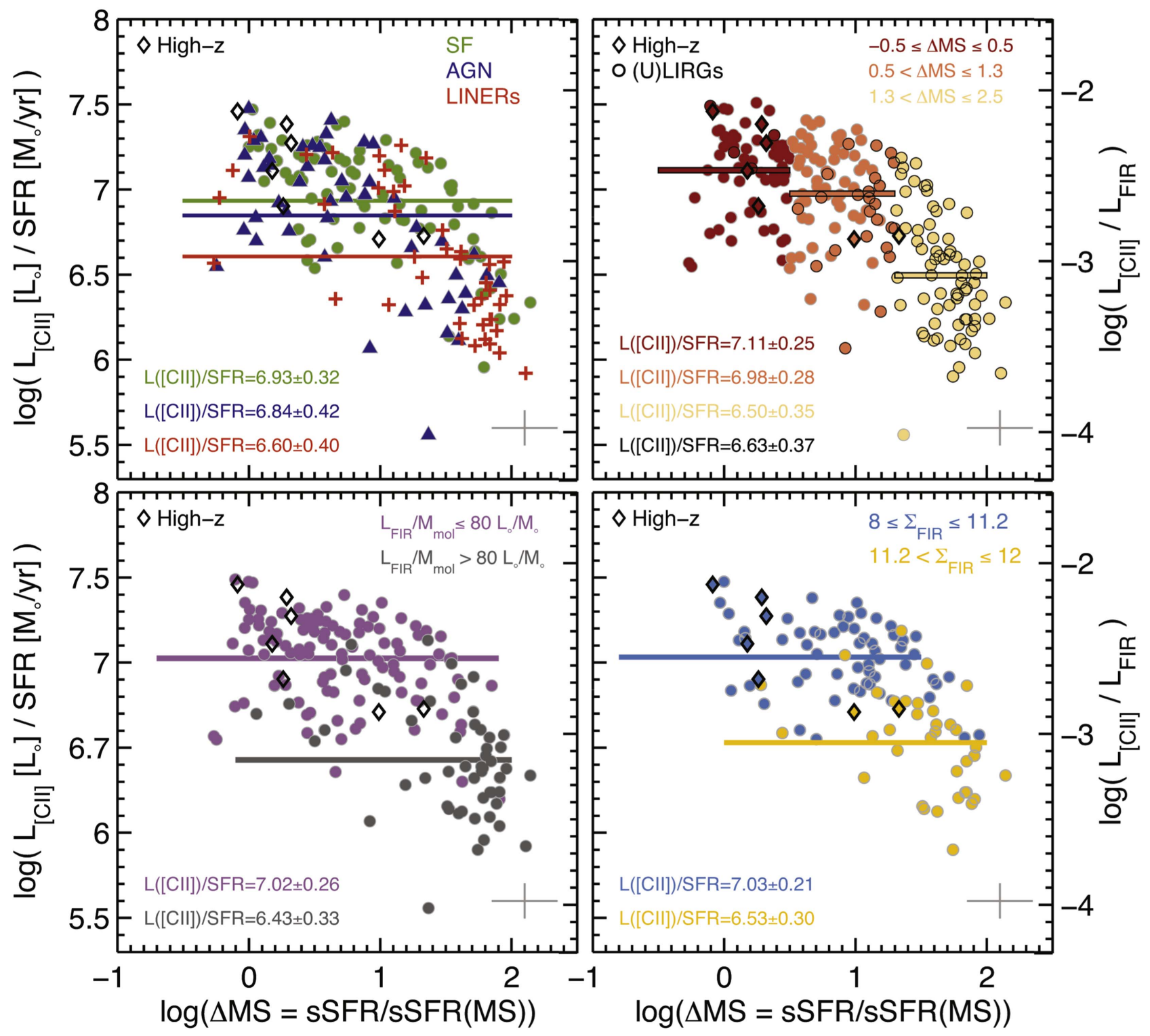

Figure 6. $L_{[\mathrm{C} \mathrm{m}}-\mathrm{SFR}$ and $\left.L_{\mathrm{[C}} \mathrm{m}\right]-L_{\mathrm{FIR}}$ ratios as a function of various galaxy properties. The mean value of the $L_{[\mathrm{C}}$ m] $/ \mathrm{SFR}$ ratio for each subgroup of galaxies is listed in the bottom-left corners of the panels and also overplotted as a horizontal color line. High- $z$ galaxies $(z>2)$ are shown as diamonds. Panels: (Top-left) Nuclear optical classification (star-forming, AGN, or LINER) according to the BPT diagram. (Top-right) Offset from the main-sequence. In this panel, we also mark galaxies classified as (U)LIRGs with a black circle. (Bottom-left) Star formation efficiency (SFE $=L_{\mathrm{FIR}} / M_{\mathrm{mol}}$ ). (Bottom-right) far-infrared surface brightness $\Sigma_{\mathrm{FIR}}$. Star formation rates were calculated based on the total far-infrared using Murphy et al. (2011) calibration. Typical error bars are shown in the lower-right corner.

luminosity of a galaxy if a measurement of the SFR is available, or to attempt to measure the SFR if the [C II] luminosity is known. Figure 6 shows the [C II] luminosity-SFR ratio of galaxies as a function of their offset from the mainsequence $\triangle \mathrm{MS}$. In the first panel, we group galaxies according to their BPT classification, i.e., star-forming (stars), AGN (Seyfert 1 and 2; triangles), and LINERs (crosses). Starforming and AGN galaxies follow similar distributions (a twodimensional two-sample Kolgomorov-Smirnov test gives a $p$-value $p<0.01$ for the likelihood that both groups of galaxies are distributed similarly), reaffirming the results from Section 3.3 that the influence of unboscured AGNs on the global [C II]-to-FIR ratio is small. Using the SFR as a predictor of the [C II] luminosity of a galaxy only based on its BPT classification yields luminosities with an uncertainty factor of 2.1 for star-forming galaxies, and 2.5 for AGNs and LINERs.
In the second panel of Figure 6, we divide galaxies into three groups according to their separation from the main sequence: main-sequence galaxies $(1 / 3 \leqslant \Delta \mathrm{MS}<3$; red circles), star-forming galaxies above the main sequence ( $3<\Delta \mathrm{MS} \leqslant 20 ;$ orange circles), and starburst outliers ( $\Delta \mathrm{MS}>20$; golden circles). As discussed at the beginning of this section, there is a trend of decreasing $L_{[\mathrm{C}}$ II] $/ \mathrm{SFR}$ ratios as $\triangle \mathrm{MS}$ increases, which implies that starburst outliers have, on average, $L_{[\mathrm{C} \text { II] }} / \mathrm{SFR}$ ratios a factor of $\sim 5$ lower than mainsequence galaxies. We find that using the position of a galaxy relative to the main-sequence provides a prediction for the [C II] luminosity with an uncertainty factor of $\sim 2$. The panel also includes galaxies classified as LIRGs (black border circles). The majority of these correspond to the group of starburst outliers and their median $L_{[\mathrm{C} \mathrm{II}]} / \mathrm{SFR}$ ratio is $4.6 \times 10^{6} L_{\odot} /\left(M_{\odot} \mathrm{yr}^{-1}\right)$, a factor of $\sim 3$ lower than the median ratio in main-sequence galaxies. 
The third panel of Figure 6 shows galaxies grouped according to their $L_{\mathrm{FIR}} / M_{\mathrm{mol}}$ ratio, which is a measure of the star formation efficiency in star-forming galaxies. We split the sample into two groups, choosing a threshold value of $L_{\mathrm{FIR}} / M_{\mathrm{mol}}=80 L_{\odot} M_{\odot}^{-1}$; the value where we start to find a decline in the fine-structure line to FIR ratio of galaxies (see also Graciá-Carpio et al. 2011). For systems with $L_{\text {FIR }} / M_{\text {mol }} \leqslant 80 L_{\odot} M_{\odot}^{-1}$ (purple circles), we observe a tight correlation ( 0.26 dex dispersion) over two orders of magnitude in $\Delta \mathrm{MS}$. This implies that if the position of the galaxy in the main-sequence plane is unknown, then information on the $L_{\mathrm{FIR}} / M_{\text {mol }}$ ratio can lead to predictions of the [C II] luminosities with an uncertainty factor of $\sim 2$.

Finally, the last panel in Figure 6 shows galaxies grouped according to their FIR surface brightness. Those with $\Sigma_{\mathrm{FIR}}$ in the $10^{8}-10^{11.2} L_{\odot} \mathrm{kpc}^{-2}$ range show the tightest correlation among all the categories previously discussed. The scatter is only 0.21 dex over two orders of magnitude in $\Delta \mathrm{MS}$. Galaxies with $\Sigma_{\text {FIR }}>10^{11.2} L_{\odot} \mathrm{kpc}^{-2}$ show a correlation with a $1 \sigma$ dispersion of 0.3 dex. The high- $z$ systems in our sample follow these trends, which suggest that the dominant physical conditions set by the compactness of the source produce similar [C II]/SFR ratios in local and high- $z$ galaxies. This also implies that the scaling relations derived based on observations of nearby galaxies - and that rely on a third parameter such as $\Delta \mathrm{MS}$ or $\Sigma_{\mathrm{FIR}}$ — can also be applied to high- $z$ galaxies.

One caveat is the AGN contribution to the IR luminosity that can lead to the overestimation of the SFR. This effect can be particularly important in ULIRGs (e.g., Veilleux et al. 2009b). Note, however, that given the similar distribution of starforming and AGN galaxies as a function of $\triangle \mathrm{MS}$ (first panel of Figure 6), the mean [C II]/SFR (or [C II]/FIR) ratios for the different subgroups of galaxies change by $\lesssim 0.1 \mathrm{dex}$ if we exclude AGN systems from the analysis.

In summary, the [C II] luminosity of a galaxy can be predicted based on its SFR (or the SFR can be calculated based on the [C II] luminosity) with different levels of uncertainty depending on which other galaxy properties are known. For a detailed list of the scaling relations between the [C II] luminosity and the SFR see Table 1. We find that the combination of the SFR and the FIR surface brightness produce the most robust predictions of [C II] luminosities, with an uncertainty of only 0.21 dex if the galaxy is in the $\Sigma_{\text {FIR }}=10^{8}-10^{11.2} L_{\odot} \mathrm{kpc}^{-2}$ range. This is consistent with the tight $(0.21 \mathrm{dex})$ correlation between $\Sigma_{[\mathrm{C} \mathrm{II}]}-\Sigma_{\mathrm{SFR}}$ observed in resolved regions of nearby, star-forming galaxies (HerreraCamus et al. 2015).

The second best alternative to predict [C II] luminosities relies on the combination of the SFR with the separation of the galaxy with respect to the main sequence, $\Delta \mathrm{MS}$, or the $L_{\mathrm{FIR}} / M_{\text {mol }}$ ratio. In these cases, [C II] luminosities can be predicted within an uncertainty of a factor of $\approx 2$. Finally, taking as an additional parameter to the SFR the emission line classification of the galaxy-i.e., SF, AGN, or LINER-does not contribute to a better prediction of the [C II] luminosity. In these cases, the scatter associated with the observed [C II]/SFR ratio is of the order of $\sim 0.35-0.4$ dex.

\subsection{Mass-Metallicity Relation of (U)LIRGs Assessed by Extinction Insensitive Metallicity Diagnostics}

The mass-metallicity relation is a well defined relationship between stellar mass and gas-phase abundance observed in galaxies up to $z \sim 3$ (e.g., Lequeux et al. 1979; Tremonti et al. 2004; Savaglio et al. 2005; Erb et al. 2006; Lee et al. 2006; Maiolino et al. 2008; Zahid et al. 2011; Steidel et al. 2014; Wuyts et al. 2014, 2016). The existence of this relation has been interpreted as a consequence of the interplay between star formation, gas outflows, and gas accretion during the evolution of galaxies. While the mass-metallicity relation holds for low-metallicity and star-forming galaxies, (U)LIRGs have been found to deviate in the sense that their gas-phase abundance-as inferred from optical nebular lines-is much lower than expected from their stellar mass (e.g., Rupke et al. 2008; Kilerci Eser et al. 2014).

The observed offset of local (U)LIRGs from the massmetallicity relation could have at least two explanations. First, as shown by theoretical models and numerical simulations (e.g., Naab et al. 2006; Montuori et al. 2010; Rupke et al. 2010; Torrey et al. 2012), tidal forces acting in merging/interacting galaxies drive low-metallicity gas from the outskirts toward the central active star-forming regions; hence the observed nuclear metallicity under-abundances and shallower metallicity gradients (Rupke et al. 2008; Kewley et al. 2010; Kilerci Eser et al. 2014). Second, the low gas metallicity inferred from the optical nebular lines may not be representative of the metallicity of the heavily obscured bulk of the gas in (U) LIRGs. Two pieces of evidence that support these hypotheses are the large dust masses found in ULIRGs, which are incompatible with the low metallicities inferred from the optical lines (e.g., Santini et al. 2010), and the $\sim 3 \times$ solar neon abundance found in the average spectrum of $27 \mathrm{PAH}-$ dominated ULIRGs by Veilleux et al. (2009b; see also Verma et al. 2003). Note, however, that both of these measurements are subject to uncertainties, and ideally one would want an independent, reliable, and extinction insensitive determination of the metallicity to validate one of the two possible scenarios.

Far-infrared fine-structure lines originating in $\mathrm{H}$ II regions can work as powerful tools to measure the gas metallicity even in highly obscured star-forming regions. Based on a grid of photoionization models, Nagao et al. (2011), FernándezOntiveros et al. (2016), and Pereira-Santaella et al. (2017) have shown that the flux line ratios of ([O III] $52 \mu \mathrm{m}$ $+[\mathrm{O}$ III $] 88 \mu \mathrm{m}) /[\mathrm{N}$ III $] 57 \mu \mathrm{m}$ or $[\mathrm{O}$ III $] 88 \mu \mathrm{m} /[\mathrm{N}$ III $] 57 \mu \mathrm{m}$ are sensitive diagnostics of the gas metallicity at $Z>0.2 Z_{\odot}$. These line ratios present some residual dependence on the ionization parameter $U$ and on the ionized gas density; however, these quantities can be constrained using a combination of far-infrared H II and PDR lines, as we have shown in Section 3.2.

In this section, we use the [N III] $57 \mu \mathrm{m}$ and [O III] $88 \mu \mathrm{m}$ lines to determine the extinction-insensitive metallicity of 11 (U)LIRGs. Eight of these systems have optical gas-phase metallicities available: four selected from SHINING, and the other four selected from the Sloan Digital Sky Survey (SDSS) and observed with Herschel/PACS (PI R. Maiolino; see Table 2 for details). These systems do not show evidence for AGN activity.

For the SDSS galaxies, stellar masses and optical metallicities were drawn from the JHU/MPA value-added galaxy catalog $^{14}$ (Kauffmann et al. 2003; Brinchmann et al. 2004; Tremonti et al. 2004). For the SHINING galaxies IRAS F10565+2448 and IRAS F08572+3915, we use oxygen

\footnotetext{
14 http://home.strw.leidenuniv.nl/ jarle/SDSS /
} 

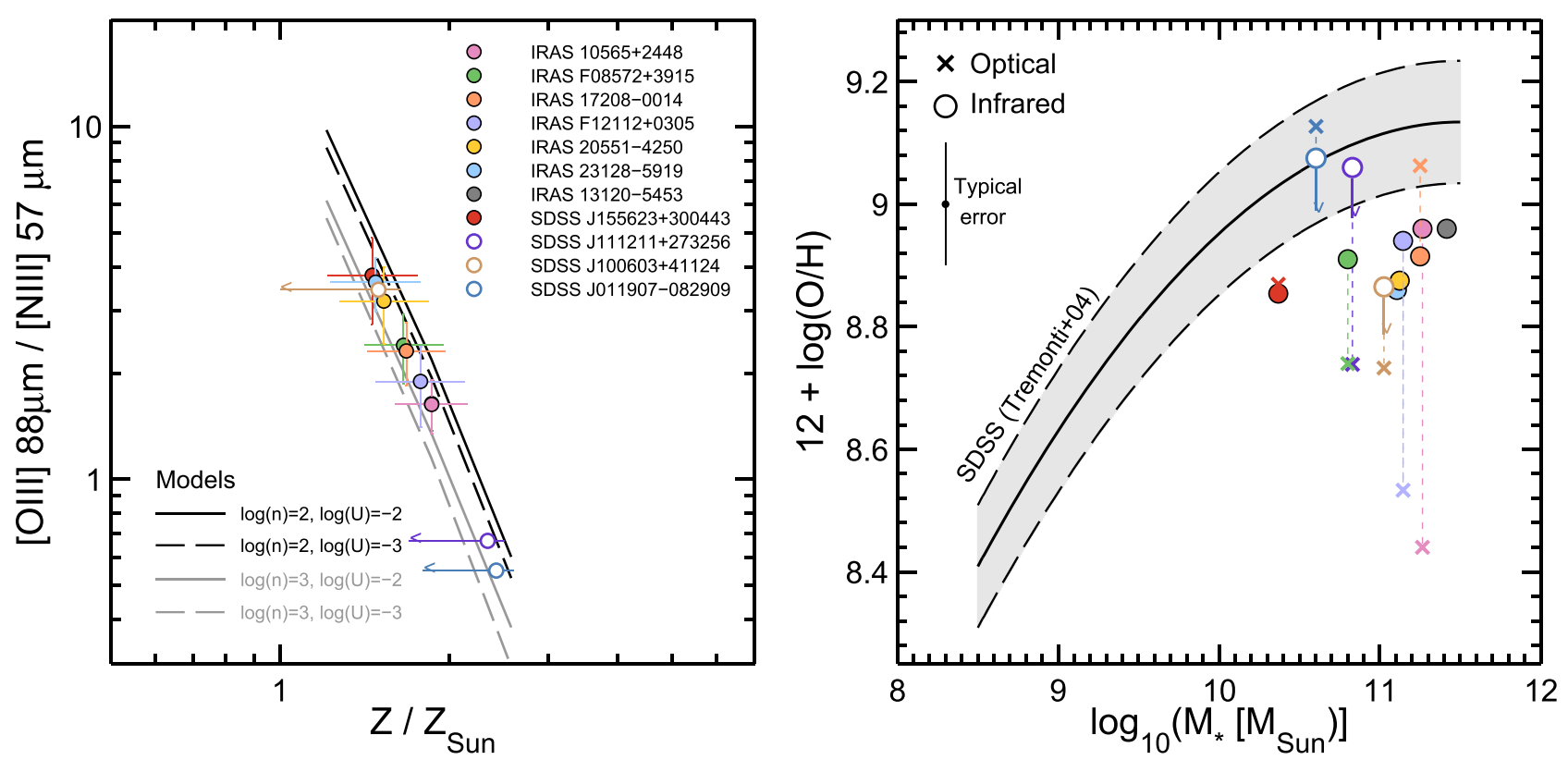

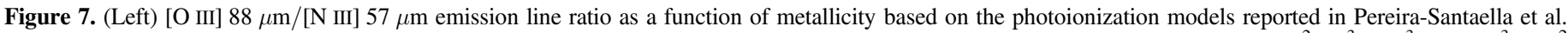

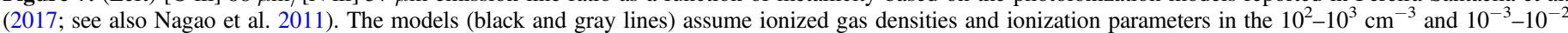

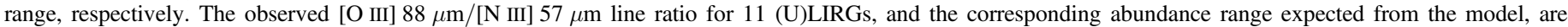

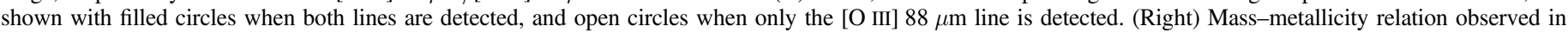

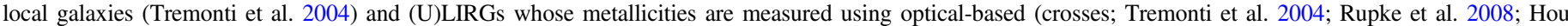

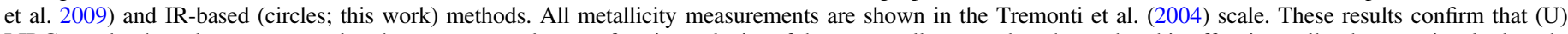

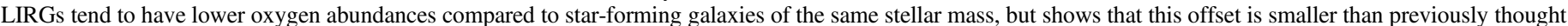
from studies based on optical-based metallicities (e.g., Rupke et al. 2008).

abundances in the Tremonti et al. (2004) calibration scale as listed in Rupke et al. (2008). For IRAS 17208-0014 and IRAS $\mathrm{F} 12112+0305$, we draw $\mathrm{H} \alpha$ and [N II] 6584 fluxes from Moustakas \& Kennicutt (2006) and Hou et al. (2009), and then derived oxygen abundances based on the $[\mathrm{N} \mathrm{II}] / \mathrm{H} \alpha$ diagnostic in Maiolino et al. (2008). We converted these metallicities to the Tremonti et al. (2004) scale using the conversion factor in Kewley \& Ellison (2008).

The left panel of Figure 7 shows the measured [O III] $88 \mu \mathrm{m} /$ [N III] $57 \mu \mathrm{m}$ line ratios (and lower-limits) as a function of metallicity based on the photoionization models reported in Pereira-Santaella et al. (2017). The model results we include are based on a range of ionization parameters and density values typical of (U)LIRGs as estimated in Section 3.2 (see also GraciáCarpio et al. 2011; Fischer et al. 2014). The model curves shown in Figure 7 have been scaled to take into account the differences in the Pereira-Santaella et al. (2017) and Tremonti et al. (2004) metallicity calibrations. While the latter uses the model of Charlot \& Longhetti (2001, hereafter CL01), the infrared method in Pereira-Santaella et al. (2017) scales the nitrogen abundance with oxygen following the fit to the observed relation in Pilyugin et al. (2014, hereafter PL14). Both models CL01 and PL14 have parameterizations of the $\mathrm{N} / \mathrm{O}$ ratio that increase as a function of $\mathrm{O} / \mathrm{H}$ at a similar rate for $Z>0.25 Z_{\odot}$, but with different normalization values. At a solar metallicity, the CLO1 model yields a $\mathrm{N} / \mathrm{O}$ abundance that is a factor of $\sim 2$ lower than the $\mathrm{N} / \mathrm{O}$ abundance in the PL14 calibration $\left(\log _{10}(\mathrm{~N} / \mathrm{O})_{\mathrm{CL} 01} \approx-0.95\right.$ versus $\left.\log _{10}(\mathrm{~N} / \mathrm{O})_{\mathrm{PL} 14} \approx-0.65\right)$. According to the scaled version of the Pereira-Santaella et al. (2017) models, the IRbased metallicities of the (U)LIRGs in our sample are in the $1.5 \lesssim Z / Z_{\odot} \lesssim 2.5$ range.

How do the IR-based metallicity measurements compare to those obtained from optical nebular lines? The right panel of
Figure 7 shows the comparison between both methods in the context of the observed mass-metallicity relation in local $(z \sim 0.1), \quad$ star-forming galaxies from SDSS (Tremonti et al. 2004). Out of the five (U)LIRGs detected in both [O III] $88 \mu \mathrm{m}$ and [N III] $57 \mu \mathrm{m}$ lines, we find that the two systems with the lowest optical metallicities-IRAS F12112+0305 and IRAS F10565+2448 - have infrared-based metallicities a factor of $\sim 3$ higher. The remaining three (U)LIRGs have optical- and infrared-based metallicities that are consistent within the error bars. For IRAS F12112+0305 and IRAS F10565+2448, this would imply that the gas-phase metallicities derived from optical and infrared methods trace different layers, in the sense that gas in the densest and dustiest star-forming regions that can only be probed by infrared lines is more enriched than the gas in less obscured regions (Veilleux et al. 2009b; Santini et al. 2010).

In summary, we find that the oxygen abundance of nonAGN (U)LIRGs derived from both optical and infrared emission line tracers tend to be lower than the metallicity of star-forming galaxies of similar stellar mass. This is in agreement with previous mass-metallicity studies of (U)LIRGs (Rupke et al. 2008; Kilerci Eser et al. 2014; Pereira-Santaella et al. 2017). Note, however, that using infrared-based metallicities reduces the offset from the mass-metallicity relation previously found using optical line diagnostics. Among the two competing hypotheses to explain the offset from the mass-metallicity relation-massive inflows of metal-poor gas or the bulk of the gas being metal-rich, but heavily embedded in dust-our comparison of optical and infrared-based metallicity measurements favors the former.

\section{Summary and Conclusions}

In this paper, we investigate the physical mechanisms behind the the observed line deficits in galaxies. We also investigate the 
scaling relations between the [C II] emission and the SFR, and the location of the LIRGs in the well-known mass-metallicity relation of star-forming galaxies. The analysis presented here is based on the SHINING survey of galaxies (Paper I; Herrera-Camus et al. 2018), which was conducted with the PACS spectrometer on board the Herschel Space Observatory.

We highlight the following points:

1. A Toy Model to explain the PDR lines deficit. To explore the tight relationship observed between the [C II] to continuum ratio and $\Sigma_{\mathrm{FIR}}$, we created a toy model that treats the ISM as the combination of two extreme scenarios. Scenario $1-$ Dense PDR: we assume that all $\mathrm{OB}$ stars and molecular gas clouds are closely associated. We also consider that radiation pressure in $\mathrm{HII}$ regions acts to concentrate the gas in a spherical shell, which results in a higher density of the neutral gas confining the ionized gas than if we assume a uniform-density H II region (Draine 2011). In this scenario, we find that the [C II]-to-FIR ratio starts to decrease at around $\Sigma_{\mathrm{FIR}} \sim 3 \times 10^{10} L_{\odot} \mathrm{kpc}^{-2}$ because at this value (1) the density of the neutral gas becomes higher than the critical density of the [C II] line for collisional excitation by $\mathrm{H}$ atoms, and (2) the ionization parameter reaches a limit value of $U \approx 0.01$ - the threshold value of $U$ at which the fraction of UV photons absorbed by dust in the H II region becomes important (Luhman et al. 2003; Graciá-Carpio et al. 2011). This implies that the [C II] intensity is only weakly dependent on $G_{0}$ and $n_{\mathrm{H}}$, while the FIR intensity remains proportional to $G_{0}$. Scenario 2-Average ISRF: we assume that $\mathrm{OB}$ associations and neutral gas clouds are randomly placed in the galactic disk. In this case, we find that at $\Sigma_{\mathrm{FIR}}$ $\sim 10^{10} L_{\odot} \mathrm{kpc}^{-2}$ the [C II]-to-FIR ratio starts to decline as a function of $\Sigma_{\mathrm{FIR}}$ because the [C II] intensity becomes nearly independent of $G_{0}$ - as opposed to the FIR intensity that remains proportional to this quantity, and the photoelectric heating efficiency decreases by two orders of magnitude.

Compared to the observations, the combination of the output from both model scenarios is successful in reproducing the decline of the [C II]-to-FIR ratio as a function of $\Sigma_{\text {FIR }}$ starting at about $\Sigma_{\text {FIR }} \approx 10^{10} L_{\odot} \mathrm{kpc}^{-2}$. This make sense, as the real structure of the ISM is porous; therefore, only a fraction of the photons produced by massive stars interacts with the surrounding dense neutral gas (Scenario 1), while the rest illuminates neutral gas clouds in the galactic disk (Scenario 2).

The threshold value of $\Sigma_{\mathrm{FIR}} \approx 3 \times 10^{10} L_{\odot} \mathrm{kpc}^{-2}$ at which our model predicts a sharper decline in the [C II]/FIR ratio is remarkably similar to the $\Sigma_{\mathrm{FIR}}$ value that differentiates galaxies forming stars in normal or starburst mode (e.g., Elbaz et al. 2011), and PDRs from having constant or increasing $G_{0} / n_{\mathrm{H}}$ ratios (Díaz-Santos et al. 2017). This indicates that the properties of the PDR/H II region complexes in these two group of galaxies are significantly different, which leads to the observed differences in their global [C II]/FIR ratios.

Our toy model is also successful at reproducing the trends observed for the [O I] $63 \mu \mathrm{m}$ and [O I] $145 \mu \mathrm{m}$ lines.

2. Cloudy modeling. In addition to the toy model calculations, we use the Cloudy-based models described in Fischer et al. (2014) and Abel et al. (2009) to study the trends observed in the line to continuum ratios of galaxies as a function of the ionization parameter $U$ and the hydrogen column density $N_{\mathrm{H}}$, for a simple shell geometry. We find that the observed decrease in the $([\mathrm{C} \mathrm{II}]+[\mathrm{O} \mathrm{I}] 63 \mu \mathrm{m}+[\mathrm{O} \mathrm{I}] 145 \mu \mathrm{m}) / \mathrm{FIR}$, [N II] $122 \mu \mathrm{m} / \mathrm{FIR}$, and [N III] $57 \mu \mathrm{m} /[\mathrm{N}$ II] $122 \mu \mathrm{m}$ ratios as the FIR surface brightness increases from $\Sigma_{\mathrm{FIR}} \sim 10^{10}$ to $10^{12} L_{\odot} \mathrm{kpc}^{-2}$ can be explained by increasing $N_{\mathrm{H}}$ from $\sim 10^{23.5}$ to $\sim 10^{24} \mathrm{~cm}^{-2}$ and $U$ from $\sim 10^{-3}$ to $\sim 10^{-2}$. These results are consistent with previous studies (e.g., GraciáCarpio et al. 2011; Fischer et al. 2014), and also the interpretation from our toy model in the scenario where $\mathrm{OB}$ stars are closely associated to molecular clouds.

3. AGN impact on the $[C \mathrm{II}]$ and $[O \mathrm{I}]$ line emission. In Seyfert galaxies that can be spatially resolved by Herschel/PACS, we find that central regions have [C II]-to-FIR ratios that are a factor of $\sim 2-6$ lower than the median ratio in the disk. In contrast, we find central [O I] $63 \mu \mathrm{m}$-to-FIR ratios that are comparable to or even higher than the median disk value. We also find very strong [O I] $63 \mu \mathrm{m}$, [O I] $145 \mu \mathrm{m}$, and [O III] $88 \mu \mathrm{m}$ emission in the central spaxel of compact H II Seyfert galaxies. This is probably a consequence of the denser gas and the harder radiation fields to which the ISM is exposed in the central AGN regions, which favors the cooling of the neutral gas via the [O I] $63 \mu \mathrm{m}$ line emission. Models of Abel et al. (2009) and Fischer et al. (2014) also show that this ratio increases for high $U$ and high density. One additional factor that can contribute to the $[\mathrm{C} \mathrm{II}]$ deficit observed in AGNs is the change in the $\mathrm{C}^{++} / \mathrm{C}^{+}$balance due to the hardness of the AGN radiation field (Langer \& Pineda 2015). The only case where we find this effect could have a significant impact on the [C II]-to-FIR ratio is in the central region of Seyfert galaxy NGC 1068.

In contrast, we find that AGN activity does not play a major role in setting the global-scale [C II] to FIR ratio in (U)LIRGs.

4. Scaling relations. One of the goals of this paper is to provide a reference sample that can be used to analyze local and high- $z$ infrared line observations of galaxies. In addition to the tables with line and continuum fluxes for the full SHINING sample listed in Paper I (HerreraCamus et al. 2018), here we present scaling relations for the [C II]-to-SFR ratio as a function of galaxy type (H II, AGNs, LINERs, and LIRGs), $L_{\mathrm{FIR}} / M_{\mathrm{mol}}, \Sigma_{\mathrm{FIR}}$, and separation from the main-sequence of galaxies $(\Delta \mathrm{MS})$. These can be useful for those interested in planning [C II] observations of local or high- $z$ galaxies, or those who want to use the [C II] line as an SFR tracer. We conclude that the most reliable method to predict [C II] fluxes-or to measure SFRs from [C II] fluxes-is when information on the infrared size of the source is available. This is likely due to the fact that normalized quantities such as $\Sigma_{\text {FIR }}$ or $\Sigma_{\text {SFR }}$ are more representative of $G_{0}$ (the local FUV radiation field intensity), one of the main parameters controlling the $[\mathrm{C} \mathrm{II}]$ relative line intensity in the neutral ISM (see also De Looze et al. 2014; Herrera-Camus et al. 2015; Smith et al. 2017).

5. Extinction-insensitive metallicity diagnostics of (U)LIRGs. We use the [O III] $88 \mu \mathrm{m} /[\mathrm{N}$ III] $57 \mu \mathrm{m}$ emission line ratio, in combination with the models by Pereira-Santaella et al. (2017; see also Nagao et al. 2011), to determine the oxygen phase-abundance of eight (U)LIRGs that, according to their optical-based metallicities, fall below the local massmetallicity relation (Tremonti et al. 2004). We find that the (U)LIRGs in our sample have infrared-based metallicities in the $1.5 \lesssim Z / Z_{\odot} \lesssim 2.5$ range. For two of the 
systems, the infrared-based metallicities are a factor of $\sim 3$ higher than the optical-based measurements. We confirm that (U)LIRGs lie below the observed local massmetallicity relation as previously reported based on optical recombination line studies (e.g., Rupke et al. 2008; Kilerci Eser et al. 2014). These results are consistent with a scenario where the oxygen under-abundance observed in (U)LIRGs is due to merger-driven massive inflows of metal-poor gas from the outskirts of the disk to central regions (Naab et al. 2006; Rupke et al. 2008; Kewley et al. 2010), but we cannot rule out (1) the effects of extinction of the most enriched regions even in the FIR and (2) that line ratios of two lines that are observed in deficit are more sensitive to the emission from the non-deficit regions (Fischer et al. 2014), i.e., the outer regions of the system.

We thank the referee for helpful and constructive comments that improved the paper. We thank Mark Wolfire, Natascha Förster Schreiber, Shmuel Bialy, and Taro Shimizu for helpful discussions and comments. R.H.C. is thankful for the support and encouragement of Fares Bravo Garrido and dedicates this paper with love to Fares and Olivia. Basic research in IR astronomy at NRL is funded by the US ONR. J.F. also acknowledges support from the NHSC. E.G.A. is a Research Associate at the Harvard-Smithsonian Center for Astrophysics, and thanks the Spanish Ministerio de Economía y Competitividad for support under projects FIS2012-39162-C0601 and ESP2015-65597-C4-1-R, and NASA grant ADAP NNX15AE56G. R.M. acknowledges the ERC Advanced Grant 695671 "QUENCH" and support from the Science and Technology Facilities Council (STFC). The Herschel spacecraft was designed, built, tested, and launched under a contract to ESA managed by the Herschel/Planck Project team by an industrial consortium under the overall responsibility of the prime contractor Thales Alenia Space (Cannes), including Astrium (Friedrichshafen), was responsible for the payload module and for system testing at spacecraft level, Thales Alenia Space (Turin) was responsible for the service module, and Astrium (Toulouse) was responsible for the telescope, in excess of a hundred subcontractors. PACS has been developed by a consortium of institutes led by MPE (Germany) and including UVIE (Austria); KU Leuven, CSL, IMEC (Belgium); CEA, LAM (France); MPIA (Germany); INAF-IFSI/OAA/OAP/OAT, LENS, SISSA (Italy); IAC (Spain). This development has been supported by the funding agencies BMVIT (Austria), ESA-PRODEX (Belgium), CEA/ CNES (France), DLR (Germany), ASI/INAF (Italy), and CICYT/ MCYT (Spain). HCSS/HSpot/HIPE is a joint development (are joint developments) by the Herschel Science Ground Segment Consortium, consisting of ESA, the NASA Herschel Science Center, and the HIFI, PACS, and SPIRE consortia. This publication makes use of data products from the Sloan Digital Sky Survey (SDSS). Funding for the Sloan Digital Sky Survey has been provided by the Alfred P. Sloan Foundation, the Participating Institutions, the National Aeronautics and Space Administration, the National Science Foundation, the U.S. Department of Energy, the Japanese Monbukagakusho, and the Max Planck Society. This research has also made use of the NASA/IPAC Extragalactic Database (NED), which is operated by the Jet Propulsion Laboratory, California Institute of Technology, under contract with the National Aeronautics and Space Administration.

Facility: Herschel (Pilbratt et al. 2010).

Software: CLOUDY (Ferland et al. 2013), HIPE (v13.0; Ott 2010).

\section{Appendix \\ [N III] $57 \mu \mathrm{m}$ and [O III] $88 \mu \mathrm{m}$ Line Observations in (U) LIRGs Selected from SDSS}

Table 2 lists the SDSS selected (U)LIRGs observed with Herschel PACS that were included in the analysis of the massmetallicity relation in section 3.5.

Table 2

(U)LIRGs Selected from SDSS

\begin{tabular}{|c|c|c|c|c|c|}
\hline Source & $\begin{array}{c}\log \left(L_{\mathrm{FIR}}\right) \\
\left(L_{\odot}\right)\end{array}$ & $\begin{array}{c}\log \left(M_{*}\right) \\
\left(M_{\odot}\right)\end{array}$ & $12+\log (\mathrm{O} / \mathrm{H})$ & $\begin{array}{l}\text { [N III] } 57 \mu \mathrm{m} \\
\left(\mathrm{W} \mathrm{m}^{-2}\right)\end{array}$ & $\begin{array}{c}{[\mathrm{O} \text { III] } 88 \mu \mathrm{m}} \\
\left(\mathrm{W} \mathrm{m}^{-2}\right)\end{array}$ \\
\hline SDSS J155623+300443 & 11.71 & 10.36 & 8.87 & $2.41 \mathrm{E}-17 \pm 5.3 \mathrm{E}-18$ & $9.14 \mathrm{E}-17 \pm 6.5 \mathrm{E}-18$ \\
\hline SDSS J011907-082909 & 12.09 & 10.60 & 9.13 & $<5.76 \mathrm{E}-17$ & $3.17 \mathrm{E}-17 \pm 2.36 \mathrm{E}-18$ \\
\hline SDSS J100603+411224 & 12.49 & 11.02 & 8.73 & $<1.28 \mathrm{e}-17$ & $4.41 \mathrm{E}-17 \pm 7 \mathrm{E}-18$ \\
\hline
\end{tabular}

Note. Stellar masses and metallicities drawn from the SDSS JHU/MPA value added catalog. 


\section{ORCID iDs}

R. Herrera-Camus (1) https://orcid.org/0000-0002-2775-0595

E. Sturm (i) https://orcid.org/0000-0002-0018-3666

J. Graciá-Carpio (10 https://orcid.org/0000-0003-4689-3134

D. Lutz (i) https://orcid.org/0000-0003-0291-9582

S. Veilleux (1) https://orcid.org/0000-0002-3158-6820

J. Fischer (i) https://orcid.org/0000-0001-6697-7808

E. González-Alfonso (10) https://orcid.org/0000-0001-5285-8517

A. Poglitsch (i) https://orcid.org/0000-0002-6414-9408

L. Tacconi (i) https://orcid.org/0000-0002-1485-9401

R. Genzel (ㄴ) https://orcid.org/0000-0002-2767-9653

A. Verma (i) https://orcid.org/0000-0002-0730-0781

\section{References}

Abel, N. P., Dudley, C., Fischer, J., Satyapal, S., \& van Hoof, P. A. M. 2009, ApJ, 701, 1147

Bakes, E. L. O., \& Tielens, A. G. G. M. 1994, ApJ, 427, 822

Baldwin, J. A., Phillips, M. M., \& Terlevich, R. 1981, PASP, 93, 5

Boselli, A., Gavazzi, G., Lequeux, J., \& Pierini, D. 2002, A\&A, 385, 454

Brauher, J. R., Dale, D. A., \& Helou, G. 2008, ApJS, 178, 280

Brinchmann, J., Charlot, S., White, S. D. M., et al. 2004, MNRAS, 351, 1151

Calzetti, D., Wu, S.-Y., Hong, S., et al. 2010, ApJ, 714, 1256

Capak, P. L., Carilli, C., Jones, G., et al. 2015, Natur, 522, 455

Cappi, M., Panessa, F., Bassani, L., et al. 2006, A\&A, 446, 459

Carniani, S., Maiolino, R., Pallottini, A., et al. 2017, A\&A, 605, A42

Charlot, S., \& Longhetti, M. 2001, MNRAS, 323, 887

Cigan, P., Young, L., Cormier, D., et al. 2016, AJ, 151, 14

Contursi, A., Baker, A. J., Berta, S., et al. 2017, A\&A, 606, A86

Contursi, A., Poglitsch, A., Grácia Carpio, J., et al. 2013, A\&A, 549, A118

Croxall, K. V., Smith, J. D., Pellegrini, E., et al. 2017, ApJ, 845, 96

Croxall, K. V., Smith, J. D., Wolfire, M. G., et al. 2012, ApJ, 747, 81

Daddi, E., Dickinson, M., Morrison, G., et al. 2007, ApJ, 670, 156

Daddi, E., Elbaz, D., Walter, F., et al. 2010, ApJL, 714, L118

De Breuck, C., Williams, R. J., Swinbank, M., et al. 2014, A\&A, 565, A59

De Looze, I., Cormier, D., Lebouteiller, V., et al. 2014, A\&A, 568, A62

de Rosa, A., Panessa, F., Bassani, L., et al. 2012, MNRAS, 420, 2087

Díaz-Santos, T., Armus, L., Charmandaris, V., et al. 2013, ApJ, 774, 68

Díaz-Santos, T., Armus, L., Charmandaris, V., et al. 2017, ApJ, 846, 32

Dopita, M. A., Groves, B. A., Sutherland, R. S., Binette, L., \& Cecil, G. 2002, ApJ, 572, 753

Draine, B. T. 2011, ApJ, 732, 100

Elbaz, D., Daddi, E., Le Borgne, D., et al. 2007, A\&A, 468, 33

Elbaz, D., Dickinson, M., Hwang, H. S., et al. 2011, A\&A, 533, A119

Erb, D. K., Shapley, A. E., Pettini, M., et al. 2006, ApJ, 644, 813

Farrah, D., Lebouteiller, V., Spoon, H. W. W., et al. 2013, ApJ, 776, 38

Ferkinhoff, C., Brisbin, D., Nikola, T., et al. 2015, ApJ, 806, 260

Ferland, G., van Hoof, P., Verner, D., et al. 1999, Cloudy: Numerical simulation of plasmas and their spectra, Astrophysics Source Code Library, ascl:9910.001

Ferland, G. J., Porter, R. L., van Hoof, P. A. M., et al. 2013, RMxAA, 49, 137 Fernández-Ontiveros, J. A., Spinoglio, L., Pereira-Santaella, M., et al. 2016, ApJS, 226, 19

Fischer, J., Abel, N. P., González-Alfonso, E., et al. 2014, ApJ, 795, 117

Förster Schreiber, N. M., Genzel, R., Lutz, D., Kunze, D., \& Sternberg, A. 2001, ApJ, 552, 544

Genzel, R., Tacconi, L. J., Gracia-Carpio, J., et al. 2010, MNRAS, 407, 2091

Genzel, R., Tacconi, L. J., Lutz, D., et al. 2015, ApJ, 800, 20

Goicoechea, J. R., Teyssier, D., Etxaluze, M., et al. 2015, ApJ, 812, 75

González-Alfonso, E., Fischer, J., Graciá-Carpio, J., et al. 2012, A\&A, 541, A4

González-Alfonso, E., Fischer, J., Sturm, E., et al. 2015, ApJ, 800, 69

González-Alfonso, E., Smith, H. A., Ashby, M. L. N., et al. 2008, ApJ, 675, 303

González-Alfonso, E., Smith, H. A., Fischer, J., \& Cernicharo, J. 2004, ApJ, 613, 247

Graciá-Carpio, J., Sturm, E., Hailey-Dunsheath, S., et al. 2011, ApJL, 728, L7 Helou, G., Khan, I. R., Malek, L., \& Boehmer, L. 1988, ApJS, 68, 151 Herrera-Camus, R., Bolatto, A., Smith, J. D., et al. 2016, ApJ, 826, 175 Herrera-Camus, R., Bolatto, A. D., Wolfire, M. G., et al. 2015, ApJ, 800, 1 Herrera-Camus, R., Sturm, E., Graciá-Carpio, J., et al. 2018, ApJ, 861, 94 Hollenbach, D. J., \& Tielens, A. G. G. M. 1999, RvMP, 71, 173

Hou, L. G., Wu, X.-B., \& Han, J. L. 2009, ApJ, 704, 789

Howell, J. H., Armus, L., Mazzarella, J. M., et al. 2010, ApJ, 715, 572
Ibar, E., Lara-López, M. A., Herrera-Camus, R., et al. 2015, MNRAS, 449, 2498 Inoue, A. K., Tamura, Y., Matsuo, H., et al. 2016, Sci, 352, 1559

Iyomoto, N., Makishima, K., Fukazawa, Y., Tashiro, M., \& Ishisaki, Y. 1997, PASJ, 49, 425

Kauffmann, G., Heckman, T. M., White, S. D. M., et al. 2003, MNRAS, 341, 33

Kaufman, M. J., Wolfire, M. G., \& Hollenbach, D. J. 2006, ApJ, 644, 283

Kaufman, M. J., Wolfire, M. G., Hollenbach, D. J., \& Luhman, M. L. 1999, ApJ, 527, 795

Kennicutt, R. C., \& Evans, N. J. 2012, ARA\&A, 50, 531

Kennicutt, R. C., Jr. 1991, in Properties of Giant H II Regions, ed. C. Leitherer et al. (Cambridge: Cambridge Univ. Press), 349

Kewley, L. J., \& Ellison, S. L. 2008, ApJ, 681, 1183

Kewley, L. J., Rupke, D., Zahid, H. J., Geller, M. J., \& Barton, E. J. 2010, ApJL, 721, L48

Kilerci Eser, E., Goto, T., \& Doi, Y. 2014, ApJ, 797, 54

Lacey, C. G., Baugh, C. M., Frenk, C. S., et al. 2008, MNRAS, 385, 1155

Langer, W. D., \& Pineda, J. L. 2015, A\&A, 580, A5

Lee, H., Skillman, E. D., Cannon, J. M., et al. 2006, ApJ, 647, 970

Lequeux, J., Peimbert, M., Rayo, J. F., Serrano, A., \& Torres-Peimbert, S. 1979, A\&A, 80, 155

Lord, S. D., Hollenbach, D. J., Haas, M. R., et al. 1996, ApJ, 465, 703

Luhman, M. L., Satyapal, S., Fischer, J., et al. 1998, ApJL, 504, L11

Luhman, M. L., Satyapal, S., Fischer, J., et al. 2003, ApJ, 594, 758

Lutz, D., Berta, S., Contursi, A., et al. 2016, A\&A, 591, A136

Lutz, D., Sturm, E., Genzel, R., et al. 2003, A\&A, 409, 867

Madden, S. C., Rémy-Ruyer, A., Galametz, M., et al. 2013, PASP, 125 , 600

Maiolino, R., Nagao, T., Grazian, A., et al. 2008, A\&A, 488, 463

Malhotra, S., Helou, G., Stacey, G., et al. 1997, ApJL, 491, L27

Malhotra, S., Kaufman, M. J., Hollenbach, D., et al. 2001, ApJ, 561, 766

Maloney, P. R., Hollenbach, D. J., \& Tielens, A. G. G. M. 1996, ApJ, 466, 561

Meijerink, R., \& Spaans, M. 2005, A\&A, 436, 397

Montuori, M., Di Matteo, P., Lehnert, M. D., Combes, F., \& Semelin, B. 2010 , A\&A, 518, A56

Moustakas, J., \& Kennicutt, R. C., Jr. 2006, ApJS, 164, 81

Muñoz, J. A., \& Oh, S. P. 2015, arXiv:1510.00397

Murphy, E. J., Condon, J. J., Schinnerer, E., et al. 2011, ApJ, 737, 67

Mushotzky, R. F., Winter, L. M., McIntosh, D. H., \& Tueller, J. 2008, ApJL, 684, L65

Naab, T., Jesseit, R., \& Burkert, A. 2006, MNRAS, 372, 839

Nagao, T., Maiolino, R., De Breuck, C., et al. 2012, A\&A, 542, L34

Nagao, T., Maiolino, R., Marconi, A., \& Matsuhara, H. 2011, A\&A, 526, A149

Narayanan, D., \& Krumholz, M. 2017, MNRAS, 467, 50

Negishi, T., Onaka, T., Chan, K.-W., \& Roellig, T. L. 2001, A\&A, 375, 566

Okada, Y., Pilleri, P., Berné, O., et al. 2013, A\&A, 553, A2

Ott, S. 2010, in ASP Conf. Ser. 434, Astronomical Data Analysis Software and Systems XIX, ed. Y. Mizumoto, K.-I. Morita, \& M. Ohishi (San Francisco, CA: ASP), 139

Parkin, T. J., Wilson, C. D., Schirm, M. R. P., et al. 2013, ApJ, 776, 65

Parravano, A., Hollenbach, D. J., \& McKee, C. F. 2003, ApJ, 584, 797

Pavesi, R., Riechers, D. A., Capak, P. L., et al. 2016, ApJ, 832, 151

Pentericci, L., Carniani, S., Castellano, M., et al. 2016, ApJL, 829, L11

Pereira-Santaella, M., Rigopoulou, D., Farrah, D., Lebouteiller, V., \& Li, J. 2017, MNRAS, 470, 1218

Pilbratt, G. L., Riedinger, J. R., Passvogel, T., et al. 2010, A\&A, 518, L1

Pilyugin, L. S., Grebel, E. K., Zinchenko, I. A., \& Kniazev, A. Y. 2014, AJ, 148,134

Pineda, J. L., Langer, W. D., \& Goldsmith, P. F. 2014, A\&A, 570, A121

Pineda, J. L., Langer, W. D., Velusamy, T., \& Goldsmith, P. F. 2013, A\&A, 554, A 103

Poglitsch, A., Herrmann, F., Genzel, R., et al. 1996, ApJL, 462, L43

Poglitsch, A., Waelkens, C., Geis, N., et al. 2010, A\&A, 518, L2+

Pound, M. W., \& Wolfire, M. G. 2008, in ASP Conf. Ser. 394, Astronomical Data Analysis Software and Systems XVII, ed. R. W. Argyle, P. S. Bunclark, \& J. R. Lewis (San Francisco, CA: ASP), 654

Rangwala, N., Maloney, P. R., Glenn, J., et al. 2011, ApJ, 743, 94

Riechers, D. A., Bradford, C. M., Clements, D. L., et al. 2013, Natur, 496, 329

Riechers, D. A., Carilli, C. L., Capak, P. L., et al. 2014, ApJ, 796, 84

Rodighiero, G., Daddi, E., Baronchelli, I., et al. 2011, ApJL, 739, L40

Röllig, M., Ossenkopf, V., Jeyakumar, S., Stutzki, J., \& Sternberg, A. 2006, A\&A, 451, 917

Rosenberg, M. J. F., van der Werf, P. P., Aalto, S., et al. 2015, ApJ, 801, 72

Rupke, D. S. N., Kewley, L. J., \& Barnes, J. E. 2010, ApJL, 710, L156

Rupke, D. S. N., Veilleux, S., \& Baker, A. J. 2008, ApJ, 674, 172

Sakamoto, K., Wang, J., Wiedner, M. C., et al. 2008, ApJ, 684, 957 
Santini, P., Maiolino, R., Magnelli, B., et al. 2010, A\&A, 518, L154

Sargsyan, L., Lebouteiller, V., Weedman, D., et al. 2012, ApJ, 755, 171

Savaglio, S., Glazebrook, K., Le Borgne, D., et al. 2005, ApJ, 635, 260

Scoville, N., Murchikova, L., Walter, F., et al. 2017, ApJ, 836, 66

Sheffer, Y., Wolfire, M. G., Hollenbach, D. J., Kaufman, M. J., \& Cordier, M. 2011, ApJ, 741, 45

Smith, J. D. T., Croxall, K., Draine, B., et al. 2017, ApJ, 834, 5

Speagle, J. S., Steinhardt, C. L., Capak, P. L., \& Silverman, J. D. 2014, ApJS, 214,15

Stacey, G. J., Hailey-Dunsheath, S., Ferkinhoff, C., et al. 2010, ApJ, 724, 957

Stacey, G. J., Viscuso, P. J., Fuller, C. E., \& Kurtz, N. T. 1985, ApJ, 289, 803

Steidel, C. C., Rudie, G. C., Strom, A. L., et al. 2014, ApJ, 795, 165

Stern, J., Laor, A., \& Baskin, A. 2014, MNRAS, 438, 901

Tacconi, L. J., Genzel, R., Saintonge, A., et al. 2018, ApJ, 853, 179

Teng, S. H., \& Veilleux, S. 2010, ApJ, 725, 1848

Torrey, P., Cox, T. J., Kewley, L., \& Hernquist, L. 2012, ApJ, 746, 108

Tremonti, C. A., Heckman, T. M., Kauffmann, G., et al. 2004, ApJ, 613, 898

U, V., Sanders, D. B., Mazzarella, J. M., et al. 2012, ApJS, 203, 9
Vasta, M., Barlow, M. J., Viti, S., Yates, J. A., \& Bell, T. A. 2010, MNRAS, 404, 1910

Veilleux, S., Kim, D.-C., Peng, C. Y., et al. 2006, ApJ, 643, 707

Veilleux, S., Kim, D.-C., Rupke, D. S. N., et al. 2009a, ApJ, 701, 587

Veilleux, S., Kim, D.-C., \& Sanders, D. B. 2002, ApJS, 143, 315

Veilleux, S., \& Osterbrock, D. E. 1987, ApJS, 63, 295

Veilleux, S., Rupke, D. S. N., Kim, D.-C., et al. 2009b, ApJS, 182, 628

Verma, A., Lutz, D., Sturm, E., et al. 2003, A\&A, 403, 829

Voit, G. M. 1992, ApJ, 399, 495

Walter, F., Decarli, R., Carilli, C., et al. 2012, Natur, 486, 233

Whitaker, K. E., van Dokkum, P. G., Brammer, G., \& Franx, M. 2012, ApJL, 754, L29

Wolfire, M. G., Hollenbach, D., \& McKee, C. F. 2010, ApJ, 716, 1191

Wolfire, M. G., Tielens, A. G. G. M., \& Hollenbach, D. 1990, ApJ, 358, 116

Wuyts, E., Kurk, J., Förster Schreiber, N. M., et al. 2014, ApJL, 789, L40

Wuyts, E., Wisnioski, E., Fossati, M., et al. 2016, ApJ, 827, 74

Zahid, H. J., Kewley, L. J., \& Bresolin, F. 2011, ApJ, 730, 137 